\title{
Assessment of the Contribution of Surface Roughness to Airframe Noise
}

\author{
Yu Liu* and Ann P. Dowling \\ University of Cambridge, Cambridge, CB2 1PZ England, United Kingdom
}

DOI: $\underline{10.2514 / 1.25217}$

\begin{abstract}
The generation of sound by turbulent boundary-layer flow at low Mach number over a rough wall is investigated by applying a theoretical model that describes the scattering of the turbulence near field into sound by roughness elements. Attention is focused on the numerical method to approximately quantify the absolute level of far-field radiated roughness noise. Models for the source statistics are obtained by scaling smooth-wall data by the increased skin friction velocity and boundary-layer thickness for a rough surface. Numerical integration is performed to determine the roughness noise, and it reproduces the spectral characteristics of the available empirical formula and experimental data. Experiments are conducted to measure the radiated sound from two rough plates in an open jet. The measured noise spectra of the rough plates are above that of a smooth plate in 1-2.5 kHz frequency and exhibit reasonable agreement with the predicted level. Estimates of the roughness noise for a Boeing 757 sized aircraft wing with idealized levels of surface roughness show that in the high-frequency region the sound radiated from surface roughness may exceed that from the trailing edge, and higher overall sound pressure levels are observed for the roughness noise. The trailing edge noise is also enhanced by surface roughness somewhat. A parametric study indicates that roughness height and roughness density significantly affect the roughness noise with roughness height having the dominant effect. The roughness noise directivity varies with different levels of surface roughness.
\end{abstract}

\section{Nomenclature}

$A=$ area of the rough wall

$A_{j} \quad=j$ th equal subarea of the rough region, $A / N_{A}$

$B=$ empirical constant for the mean velocity profile on a rough wall; Eq. (29)

$C_{A}=$ A-weighting factor

$C_{F}=$ overall skin friction coefficient, $\frac{1}{L} \int_{0}^{L} c_{f} \mathrm{~d} x_{1}$

$c \quad=$ speed of sound in free field

$c_{f}=$ local skin friction coefficient, $\tau_{w} / \frac{1}{2} \rho_{0} U^{2}$

$c_{f 0}=$ local skin friction coefficient at a distance $x_{\mathrm{le}}$ from the leading edge

$D \quad=$ directivity function

$f \quad=$ frequency

$G \quad=$ Green function

$g \quad=$ function of Strouhal number $S h^{*} ;$ Eq. (44)

$H=$ aircraft height to the ground

$I_{1}-I_{3}=$ integrals with respect to the polar coordinates $\kappa$ and $\alpha$ of the wave number plane

$J_{1} \quad=$ Bessel function of order unity

$k_{s}=$ equivalent roughness height

$k_{0}=$ acoustic wave number, $\omega / c$

$L=$ length of the flat plate

$L_{c} \quad=$ equivalent chord of an aircraft wing

$L_{s} \quad=$ equivalent span of an aircraft wing

$M \quad=$ freestream Mach number, $U / c$

$N=$ average number of roughness bosses per unit area

$N_{A}=$ number of subareas in the rough region

$n=$ empirical coefficient in $\Phi_{R}$; Eq. (35)

Presented as Paper 2510 at the 12th AIAA/CEAS Aeroacoustics Conference (27th AIAA Aeroacoustics Conference), Cambridge, MA, 8-10 May 2006; received 26 May 2006; revision received 30 November 2006; accepted for publication 5 January 2007. Copyright $@ 2007$ by the American Institute of Aeronautics and Astronautics, Inc. All rights reserved. Copies of this paper may be made for personal or internal use, on condition that the copier pay the $\$ 10.00$ per-copy fee to the Copyright Clearance Center, Inc., 222 Rosewood Drive, Danvers, MA 01923; include the code 0001-1452/07 $\$ 10.00$ in correspondence with the CCC.

${ }^{*} \mathrm{Ph}$.D. Student, Department of Engineering. Student Member AIAA.

${ }^{\dagger}$ Professor of Mechanical Engineering and Head of the Division of Energy, Fluid Mechanics and Turbomachinery, Department of Engineering. Senior Member AIAA. $\tilde{\boldsymbol{n}} \quad=\quad$ unit observer vector

$P_{R} \quad=$ acoustic frequency spectrum of far-field radiated roughness noise

$P_{s} \quad=$ smooth-wall wave-number-frequency spectrum

$p=$ pressure fluctuation

$p_{s}=$ hypothetical smooth-wall pressure fluctuation

$Q \quad=$ turbulence Reynolds stress source

$R \quad=$ roughness height

$R e_{L}=$ Reynolds number based on the whole plate, $U L / \nu$

$R e_{\mathrm{le}}=$ Reynolds number based on the distance between the rough region and leading edge, $U x_{\mathrm{le}} / \nu$

$R e_{\tau}=$ roughness Reynolds number, $k_{s} u_{\tau} / v$

$S=$ cross-spectral density of the turbulence Reynolds stress

$S h^{*}=$ Strouhal number based on the displacement boundarylayer thickness, $\omega \delta^{*} / U$

$t=$ observer time

$U=$ freestream velocity

$U_{c} \quad=$ eddy convection velocity

$u=$ streamwise mean velocity

$u_{\tau} \quad=$ friction velocity

$v=x_{2}$ component of the perturbation velocity

$\boldsymbol{v} \quad=$ perturbation velocity

$w \quad=$ wake function

$\boldsymbol{x}=$ observer position

$x_{\mathrm{le}}=$ distance between the rough region and the leading edge

$x_{0}=$ hypothetical extension of the rough region

$\boldsymbol{Y}=$ velocity potential of an ideal incompressible flow over the rough wall

$\boldsymbol{y}=$ source position

$\alpha=$ polar angle of the wave number plane

$\beta=$ empirical coefficient in $\Phi_{R}$; Eq. (35)

$\gamma \quad=\left(k_{0}^{2}-\kappa^{2}\right)^{\frac{1}{2}}$

$\Delta \quad=$ space separation vector

$\Delta t=$ difference in observer time; Eq. (49)

$\delta=$ local boundary-layer thickness

$\delta(\boldsymbol{x})=$ Dirac delta function

$\delta^{*} \quad=$ displacement boundary-layer thickness

$\delta_{0}=$ boundary-layer thickness at the front edge of the rough region

$\varepsilon \quad=$ power law exponent for OASPL variation with $R$

$\theta, \phi \quad=$ directivity angles

$\kappa=$ amplitude of the wave number $\kappa$ 


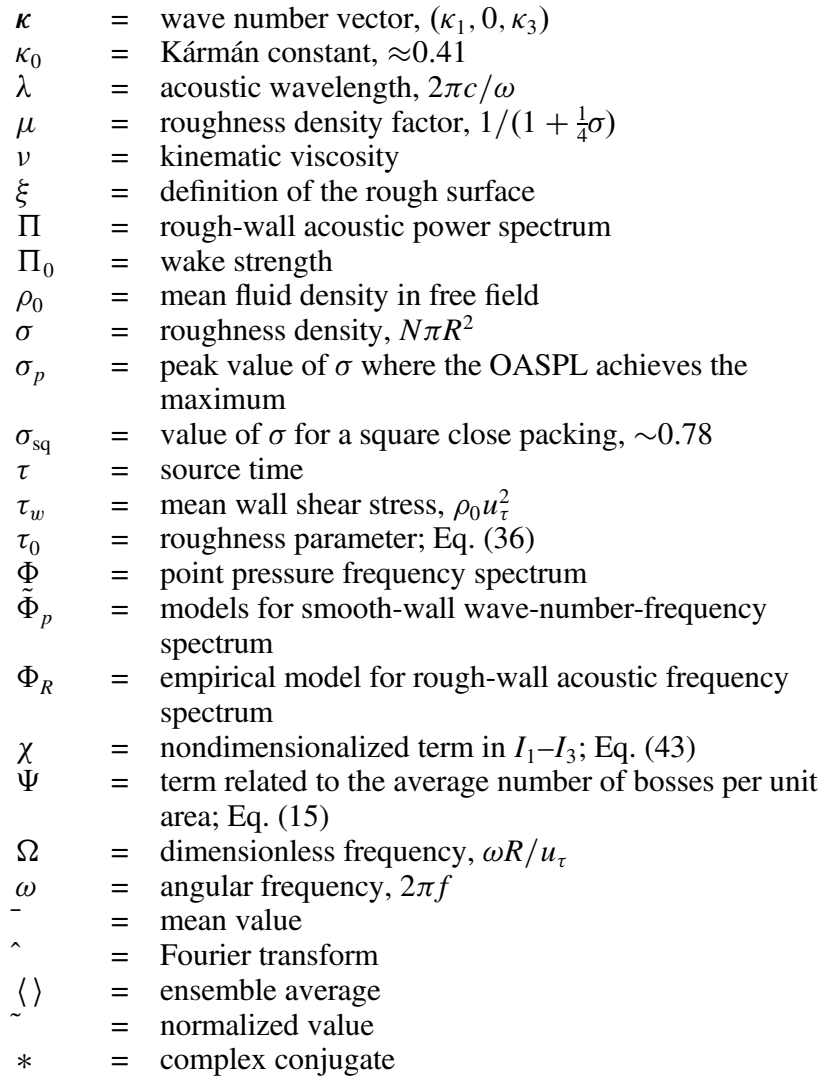

Subscripts

$\begin{array}{ll}i, j & =\text { general summation variable } \\ m & =\text { summation variable over the roughness bosses } \\ \max & =\text { maximum value } \\ \mathrm{rms} & =\text { root mean square } \\ \eta & =\text { summation variable over } x_{1} \text { and } x_{3} \text { in plane directions }\end{array}$

\section{Introduction}

$\mathbf{R}$ ECENT progress has been made in reducing engine and airframe noise. Estimates of the trailing-edge (TE) noise are low [1] and this means increasing interest in other features that may contribute to the noise of a clean configuration. Panel vibration may give noticeable noise levels [2]. In this paper we look at another mechanism whereby energy in the turbulent boundary layer may scatter into radiated sound due to the presence of small surface roughness. This might arise from discontinuities (rivets, ribs, joints, etc.), environmental contamination, paint finishes, or imprecise machining during manufacture. Relatively small amounts of surface roughness can produce substantial increases in drag compared to that predicted for the corresponding perfectly smooth boundary [3]. Similarly large increases will therefore be expected to occur in the strength of the boundary-layer generated noise.

Howe [4] has previously presented a theoretical model on the generation of sound by turbulent boundary-layer flow over a rough wall, and speculated that the surface roughness generated noise would be a substantial fraction of the airframe noise of an airplane flying in the clean configuration. In this theory, the rough surface is simply modeled by randomly distributing rigid, hemispherical bosses over an otherwise smooth plane. It is assumed that the turbulent boundary-layer roughness noise is produced primarily by the diffraction of the turbulent hydrodynamic near field (pseudosound) by the roughness bosses. The analysis is according to the classical, ideal fluid diffraction theory so that the viscous

\footnotetext{
$\$$ "Clean” means that landing gears are stowed and without slats, and thus represents how future aircraft with high-lift wings might be configured during approach.
}

stresses on the wall can be disregarded. This approximation is likely to be adequate for the surface roughness Reynolds number $R e_{\tau}=k_{s} u_{\tau} / \nu>10$, where $k_{s}$ is determined by fitting a conventional logarithmic curve to the mean boundary-layer velocity profile [3]. In this case the roughness bosses protrude beyond the viscous sublayer, and this is consistent with the usual criteria (namely, $R e_{\tau}>5$ ) for a surface to be hydraulically rough.

Howe [5] then extended the inviscid diffraction theory in [4] to ascertain the possible influence of viscous wall stresses on the diffraction mechanism. In these circumstances the roughness elements are assumed to be sufficiently small and contained entirely within the viscous sublayer (i.e., $R e_{\tau}<5$ ) that the viscous "no-slip" condition at the wall may be applied iteratively on the mean plane of the wall. By this means it is concluded that over the whole frequency range in which roughness noise is expected to be significant, viscous effects increase the levels of the radiated sound by 2 or $3 \mathrm{~dB}$ at the most.

An important limitation of the aforementioned theoretical approaches $[4,5]$ lies in the fact that they are unable to account for local effects such as the formation of wakes (vortex shedding) by the roughness elements, which creates new noise sources during the interaction of the turbulence with the wall. It is therefore assumed by Howe [4] that all of the significant turbulent pressure sources lie above the roughness elements, and it is anticipated that the diffraction theory is only likely to be adequate for surfaces whose roughness elements do not penetrate beyond the buffer zone into the fully developed turbulent region of the flow. In this case the acoustic frequency spectrum $P_{R}(\boldsymbol{x}, \omega)$ of the far-field radiated roughness noise can be expressed as an infinite integral in terms of the smoothwall wave-number-frequency spectrum $P_{s}(\kappa, \omega)$ of the hydrodynamic pressure fluctuations on a control surface located at a distance $R$ from the mean wall plane.

There have been a number of models already published for the smooth-wall wave-number-frequency spectrum. Graham [6] discussed the different models $\tilde{\Phi}_{p}(\boldsymbol{\kappa}, \omega)$ presented by Corcos [7], Efimtsov [8], Smol'yakov and Tkachenko [9], Ffowcs Williams $[10]$, and Chase $[\underline{11}, 12]$. These models can be related to $P_{s}(\boldsymbol{\kappa}, \omega)$ and expressed in terms of the friction velocity $u_{\tau}$, boundary-layer thickness $\delta$, and eddy convection velocity $U_{c}$, etc. For moderately rough surfaces, we make the same assumption as Howe [4] that the principal differences in the features of the wall pressure spectrum from those of the wall pressure spectrum on a smooth wall are accounted for by the differences in $u_{\tau}$ and $\delta$. This is expected to be a good approximation, especially in the vicinity of the convective peak [11]. Therefore in this approach the rough-wall wave-numberfrequency spectrum may be approximated by a smooth-wall formula, provided that $u_{\tau}$ and $\delta$ are increased to compensate for the enhanced surface drag and turbulence production [3] .

The integral of $P_{R}(\boldsymbol{x}, \omega)$ was conventionally evaluated by means of asymptotic approximation $[\underline{4}, \underline{13}]$ based on the wall pressure spectrum being sharply peaked in the vicinity of the convective ridge. An empirical formula for $P_{R}(\boldsymbol{x}, \omega)$ was also proposed by Howe [14] and the values of adjustable coefficients were partially estimated using the experimental data of Hersh [15] on the sound radiation by sand-roughened pipes of various grit sizes. However, not all the coefficient values have been determined yet due to insufficient experimental data; hence the absolute level of $P_{R}(x, \omega)$ has been unable to be determined so far. Therefore, the primary objective of this paper is to find a reliable solution scheme to approximately predict the far-field radiated roughness noise both in the spectral shape and in the absolute level, and then apply it to the assessment of the contribution of surface roughness to airframe noise.

The paper commences with a formulation of the diffraction theory for a rigid wall with hemispherical roughness bosses in Sec. II. This is based on the theoretical model of Howe [4], and the major derivations are presented succinctly for the sake of completeness. The acoustic pressure frequency spectrum $P_{R}(\boldsymbol{x}, \omega)$ is derived as an infinite double integral and the final expression takes the form of a weighted integral over the smooth-wall model $\tilde{\Phi}_{p}(\kappa, \omega)$. The 
increased values of $u_{\tau}$ and $\delta$ for a rough wall are determined by means of a skin friction formula [16]. In Sec. III a generally more accurate method is adopted to evaluate $P_{R} \overline{(x, \omega)}$ by direct numerical integration in the wave number space and compared with Howe's empirical model [14]. Section IV presents experimental results to validate this numerical method. Acoustic measurements were conducted to enable the comparison between the measured and predicted roughness noise spectra. Following the experimental validation, the numerical method is employed in Sec. $\mathrm{V}$ to estimate the far-field radiated roughness noise for an aircraft wing with different idealized levels of surface roughness, and the relative levels of the roughness noise and TE noise are discussed and explained. A parametric study and a directivity study are also carried out to investigate more features of the roughness generated noise. The conclusions of the investigation are then summarized in Sec. VI.

\section{Diffraction Theory of Turbulent Boundary-Layer Roughness Noise}

\section{A. Formulation of the Diffraction Problem}

The general idea of the diffraction of roughness generated sound is introduced by considering turbulent boundary-layer flow over a rough, rigid wall defined by the surface $x_{2}=\xi\left(x_{\eta}\right)$, where Greek suffix $\eta$ varies over the 1-direction and 3-direction parallel to the mean plane of the wall. The rough wall is formed by a random distribution ( $N$ per unit area) of hemispherical bosses of radius $R$ over the plane $x_{2}=0$ (see Fig. 1), and the fluid occupies the region $x_{2}>\xi\left(x_{\eta}\right)$. The mean flow is in the positive $x_{1}$ direction, and is of uniform mean density $\rho_{0}$ and sufficiently low Mach number that pressure fluctuations $p$ are related to turbulent fluctuations in the Reynolds stress $\rho_{0} v_{i} v_{j}$ by Lighthill's equation in the reduced form:

$$
\left(\frac{1}{c^{2}} \frac{\partial^{2}}{\partial t^{2}}-\nabla^{2}\right) p=Q(\boldsymbol{x}, t)
$$

where $Q(x, t)$ is the turbulence quadrupole source,

$$
Q(\boldsymbol{x}, t)=\frac{\partial^{2}\left(\rho_{0} v_{i} v_{j}\right)}{\partial x_{i} \partial x_{j}}
$$

It is implicitly assumed that the dominant Reynolds stress fluctuations occur in the region $x_{2}>R$ above the roughness elements.

Equation (1) is to be solved for the pressure in terms of the Reynolds stress $\rho_{0} v_{i} v_{j}$, subject to the inviscid, high-Reynoldsnumber, rigid boundary condition

$$
\partial p / \partial x_{n}=0 \quad \text { on } \quad x_{2}=\xi\left(x_{\eta}\right)
$$

where $x_{n}$ is a local coordinate normal to the rough wall. In application, the roughness elements generally protrude beyond the viscous sublayer at the wall into the fully turbulent region of the boundary layer. Even when this is not the case, however, it appears that only a small error is incurred by using inviscid boundary conditions at the wall [5]. To solve Eq. (1), a Green function $G(\boldsymbol{x}, \boldsymbol{y}, t, \tau)$ is introduced, which is the solution of Eq. (1) and boundary condition (3), with outgoing wave behavior when the source term $Q$ is replaced by the impulsive point source

$$
\delta(\boldsymbol{x}-\boldsymbol{y}) \delta(t-\tau), \quad x_{2}, y_{2}>\xi\left(x_{\eta}\right)
$$

It is assumed that turbulence of low Mach number generates sound whose characteristic wavelength greatly exceeds the boundary-layer thickness $\delta$ and therefore the roughness height $R$. Howe [4] determined an approximate Green function that satisfies the boundary condition $\partial G / \partial n=0$ on an infinite number of hemispherical bosses of radius $R$, distributed randomly over the plane $y_{2}=0$. In these circumstances he gave the Green function in the form

$$
G(\boldsymbol{x}, \boldsymbol{y}, t, \tau)=\frac{\delta(t-\tau-|\boldsymbol{x}-\boldsymbol{Y}| / c)}{2 \pi|\boldsymbol{x}-\boldsymbol{Y}|}
$$

provided that the far-field point $\boldsymbol{x}$ is situated at a distance much greater than $R$ from the center of the nearest boss, that is, attention is confined to components of the diffracted field whose length scales are much greater than $R$. In this formula $\boldsymbol{Y}$ is defined by

$$
\left\{\begin{array}{l}
Y_{2}=y_{2} \\
Y_{\eta}=y_{\eta}+\sum_{m} \mu R^{3}\left(y_{\eta}-x_{m \eta}\right) / 2\left|\boldsymbol{y}-\boldsymbol{x}_{m}\right|^{3}, \quad(\eta=1,3)
\end{array}\right.
$$

where $\boldsymbol{x}_{m}=\left(x_{m 1}, 0, x_{m 3}\right)$ is the center of the $m$ th boss on the plane. The factor $\mu$ is to take approximate account of mutual interactions between neighboring bosses. It is not appreciably different from unity, and is given (to within a relative error of about 4\%) by

$$
\mu=1 /\left(1+\frac{1}{4} \sigma\right)
$$

where $\sigma=N \pi R^{2}$ is the surface roughness density, that is, the fractional area of the plane covered by roughness bosses. The function $Y_{\eta}(\boldsymbol{y})$ is identical with the velocity potential describing an ideal incompressible flow in the $\eta$ direction over the rough wall. It satisfies $\partial Y_{\eta} / \partial y_{n}=0$ on $y_{2}=\xi\left(y_{\eta}\right)$ and ensures that $G$ satisfies $\partial G / \partial n=0$ when the acoustic wavelength is much larger than $R$.

Because $p$ and $G$ both satisfy the vanishing normal derivative condition (3), it follows by routine applications of the Green's theorem that the acoustic pressure $p(x, t)$ can be related to the quadrupole source $Q(\boldsymbol{y}, \tau)$ by

$$
p(\boldsymbol{x}, t)=\iint_{-\infty}^{\infty} G(\boldsymbol{x}, \boldsymbol{y}, t, \tau) Q(\boldsymbol{y}, \tau) \mathrm{d}^{3} \boldsymbol{y} \mathrm{d} \tau
$$

\section{B. Acoustic Spectrum of the Radiated Sound}

The far-field acoustic frequency spectrum will be derived concisely. A different Fourier transform from that of Howe $[\underline{4}, \underline{13}]$ is introduced according to the reciprocal relations:

$$
\begin{gathered}
\hat{f}\left(x_{2}, \boldsymbol{\kappa}, \omega\right)=\iint_{-\infty}^{\infty} f(\boldsymbol{x}, t) e^{-i \boldsymbol{\kappa} \cdot \boldsymbol{x}+i \omega t} \mathrm{~d} x_{1} \mathrm{~d} x_{3} \mathrm{~d} t \\
\boldsymbol{\kappa}=\left(\kappa_{1}, 0, \kappa_{3}\right)
\end{gathered}
$$

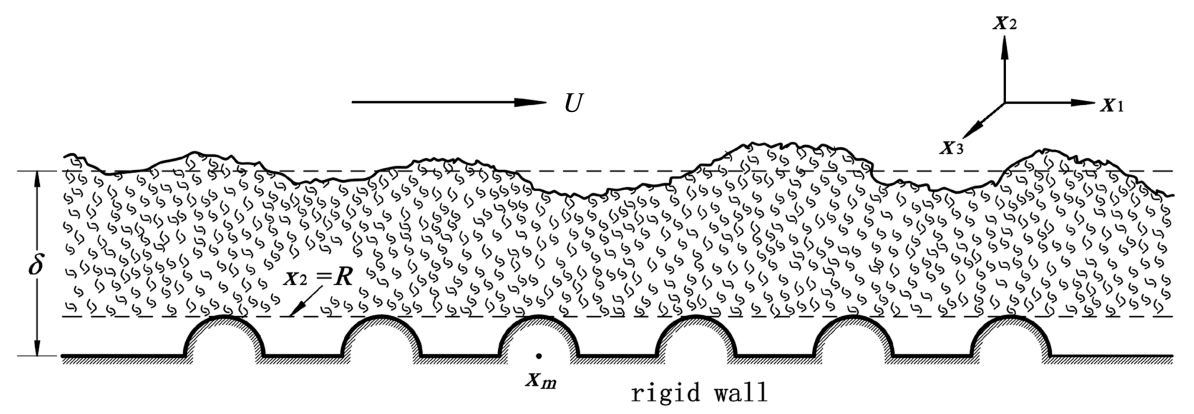

Fig. 1 Schematic illustration of the diffraction problem. 


$$
f(\boldsymbol{x}, t)=\frac{1}{(2 \pi)^{3}} \iint_{-\infty}^{\infty} \hat{f}\left(x_{2}, \kappa, \omega\right) e^{i \kappa \cdot x-i \omega t} \mathrm{~d}^{2} \kappa \mathrm{d} \omega
$$

This ensures that the Fourier transform (9) is consistent with that of the wall pressure spectrum models [] ], and Howe's derivation should be modified accordingly.

In the far field the acoustic power radiated from unit area of the rough wall is equal to the mean acoustic intensity flow $\langle p v\rangle$. The $x_{2}$ component of the perturbation velocity $v$ is determined from Eq. (8) by making use of the low Mach number approximation to the linearized momentum equation:

$$
\rho_{0} \partial v / \partial t=-\partial p / \partial x_{2}
$$

Following Howe [4], the rough-wall acoustic power spectrum $\Pi(\omega)$ is defined as the Fourier transform of $\langle p v\rangle$ with respect to $t$,

$$
\Pi(\omega)=\int_{-\infty}^{\infty}\langle p(\boldsymbol{x}, \tau) v(\boldsymbol{x}, \tau+t)\rangle e^{i \omega t} \mathrm{~d} t
$$

which expresses the frequency spectrum of the total radiated sound power per unit area of the rough wall. From Eqs. (ㅁ-ㅇ), (10), and $(11), \Pi(\omega)$ can be expressed as

$$
\begin{aligned}
& \Pi(\omega)=\frac{N \pi^{2} \mu^{2} R^{6} \omega^{2}}{6 \rho_{0} c^{3}} \int_{-\infty}^{\infty} \Psi(\kappa) e^{-2 \kappa R} \\
& \times \iint_{0}^{\infty} S\left(y_{2}, y_{2}^{\prime} ; \kappa, \omega\right) e^{i\left(\gamma y_{2}-\gamma^{*} y_{2}^{\prime}\right)} \mathrm{d} y_{2} \mathrm{~d} y_{2}^{\prime} \mathrm{d}^{2} \kappa
\end{aligned}
$$

The term $\gamma(\kappa)=\left(k_{0}^{2}-\kappa^{2}\right)^{\frac{1}{2}}$ with $k_{0}=\omega / c$, and the branch cuts are chosen such that $\operatorname{sgn}(\gamma)=\operatorname{sgn}\left(k_{0}\right)$ when $\gamma$ is real, and $\gamma \rightarrow+i \kappa$ as $|\kappa|=\kappa \rightarrow \infty$ on the real axis. $S$ is the cross-spectral density of the turbulence Reynolds stress according to the definition

$$
S\left(y_{2}, y_{2}^{\prime} ; \boldsymbol{\kappa}, \omega\right)=\frac{1}{(2 \pi)^{3}} \iint_{-\infty}^{\infty}\left\langle\hat{Q}\left(y_{2}, \boldsymbol{\kappa}, \omega\right) \hat{Q}^{*}\left(y_{2}^{\prime}, \boldsymbol{\kappa}^{\prime}, \omega^{\prime}\right)\right\rangle \mathrm{d}^{2} \boldsymbol{\kappa}^{\prime} \mathrm{d} \omega^{\prime}
$$

where $\hat{Q}\left(y_{2}, \kappa, \omega\right)$ is the Fourier transform of the Reynolds stress source $Q(\boldsymbol{y}, \tau)$. Note that $\hat{Q}\left(y_{2}, \boldsymbol{\kappa}, \omega\right)$ is well defined only for $y_{2}>R$, and it vanishes in the region occupied by the roughness bosses. In addition the term $\Psi(\kappa)$ is determined by the average number of roughness bosses in unit area of the plane. The precise value of $\Psi(\kappa)$ is unknown, but for practical purposes use may be made of an interpolation formula given by Howe $[\underline{4}, \underline{13}]$, namely,

$$
\Psi(\kappa)=\frac{\left[1-\sigma J_{1}(2 \kappa R) / \kappa R\right]^{3}}{\left[1+\sigma J_{1}(2 \kappa R) / \kappa R\right]}
$$

where $J_{1}$ is the Bessel function of order unity. This result is exact for $\kappa R=0$, and varies significantly only when $\kappa R$ exceeds unity. Possible discrepancies between interpolated and exact values for large values of $\kappa R$ are of no practical importance because of the accompanying exponential factor $e^{-2 \kappa R}$ in Eq. (12).

Let $P_{R}(\boldsymbol{x}, \omega)$ denote the frequency spectrum of the acoustic pressure fluctuations produced by diffraction by a finite area $A$ of the rough wall, defined such that

$$
\left\langle p^{2}(\boldsymbol{x}, t)\right\rangle=\frac{1}{2 \pi} \int_{-\infty}^{\infty} P_{R}(\boldsymbol{x}, \omega) \mathrm{d} \omega
$$

If the origin of coordinates is taken at the center of the region $A$, and the observer $\boldsymbol{x}$ is in the acoustic far field from $A$ in a direction specified by the polar angles $(\theta, \phi)$, as illustrated in Fig. 2, it follows from Eqs. (11), (12), and (15) that []

$$
\begin{gathered}
P_{R}(\boldsymbol{x}, \omega)=\frac{A N \pi \mu^{2} R^{6} k_{0}^{2}}{4|\boldsymbol{x}|^{2}} \int_{-\infty}^{\infty} \frac{(\boldsymbol{\kappa} \cdot \tilde{\boldsymbol{n}})^{2} \Psi(\kappa) e^{-2 \kappa R}}{\kappa^{2}} \\
\times \iint_{0}^{\infty} S\left(y_{2}, y_{2}^{\prime} ; \boldsymbol{\kappa}, \omega\right) e^{i\left(\gamma y_{2}-\gamma^{*} y_{2}^{\prime}\right)} \mathrm{d} y_{2} \mathrm{~d} y_{2}^{\prime} \mathrm{d}^{2} \kappa
\end{gathered}
$$

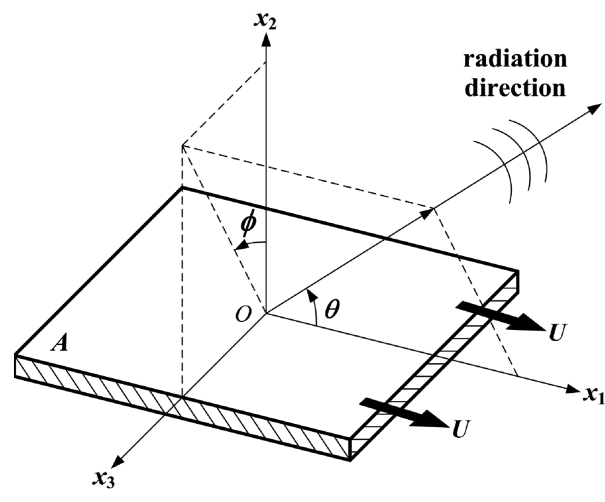

Fig. 2 Sound radiation from a region of area $A$ of the rough wall.

where $\tilde{\boldsymbol{n}}=\boldsymbol{x} /|\boldsymbol{x}|$, and

$$
\begin{gathered}
\boldsymbol{x}=|\boldsymbol{x}|(\cos \theta, \sin \theta \cos \phi, \sin \theta \sin \phi), \\
(0 \leq \theta \leq \pi,|\phi| \leq \pi / 2)
\end{gathered}
$$

is the polar representation of the far-field observer $x$. In this formula, $P_{R}(x, \omega)$ expresses the frequency spectrum of the acoustic pressure at $\boldsymbol{x}$ which is radiated within the solid angle element $\sin \theta \mathrm{d} \theta \mathrm{d} \phi$.

\section{Modeling Turbulence Reynolds Stress Sources}

To complete the determination of the acoustic frequency spectrum $P_{R}(\boldsymbol{x}, \omega)$, it is necessary to model the turbulence Reynolds stress source term, $Q(\boldsymbol{y}, \tau)$. The experimental data of Blake [17] and Schultz and Flack [18] have shown $v_{\mathrm{rms}} / u_{\tau}$ as the same function of $y / \delta$ for different surface roughness and smooth walls. We therefore assume that the source $Q(\boldsymbol{y}, \tau)$ scales as $u_{\tau}^{2}$ when expressed as a function of $y / \delta$. To implement this scaling it is convenient to introduce a hypothetical smooth-wall pressure fluctuation $p_{s}(\boldsymbol{x}, t)$ which would be generated on a smooth wall by the same Reynolds stress source. It is determined by solving Eq. (1) with the boundary condition

$$
\partial p_{s} / \partial x_{2}=0 \quad \text { on } \quad x_{2}=0
$$

and its Fourier transform is given by [19]

$$
\hat{p}_{s}(0, \kappa, \omega)=-\frac{1}{i \gamma(\kappa)} \int_{0}^{\infty} \hat{Q}\left(y_{2}, \kappa, \omega\right) e^{i \gamma y_{2}} \mathrm{~d} y_{2}
$$

The conventional wave-number-frequency spectrum $P_{s}(\kappa, \omega)$ of a smooth wall is the Fourier transform of the cross correlation of the pressure in the plane of the wall,

$$
\left\langle\hat{p}_{s}(0, \boldsymbol{\kappa}, \omega) \hat{p}_{s}^{*}\left(0, \boldsymbol{\kappa}^{\prime}, \omega^{\prime}\right)\right\rangle=(2 \pi)^{3} P_{s}(\boldsymbol{\kappa}, \omega) \delta\left(\kappa-\boldsymbol{\kappa}^{\prime}\right) \delta\left(\omega-\omega^{\prime}\right)
$$

For a rough wall it is necessary to relocate the plane of definition to be $x_{2}=R+0$, just above the roughness bosses. By hypothesis, $Q$ vanishes for $y_{2}<R$, that is, the principal Reynolds stress noise sources are assumed to be confined to the region $y_{2}>R$. The substitution of Eq. (19) into Eq. (20) gives

$$
P_{s}(\boldsymbol{\kappa}, \omega)=\frac{1}{|\gamma(\kappa)|^{2}} \iint_{0}^{\infty} S\left(y_{2}, y_{2}^{\prime} ; \kappa, \omega\right) e^{i\left(\gamma y_{2}-\gamma^{*} y_{2}^{\prime}\right)} \mathrm{d} y_{2} \mathrm{~d} y_{2}^{\prime}
$$

where the Reynolds stress cross-spectral density $S\left(y_{2}, y_{2}^{\prime} ; \boldsymbol{\kappa}, \omega\right)$, as defined in Eq. (13), is highly peaked in the neighborhood of the convective ridge of wave-number-frequency space. Substituting Eq. (21) into Eq. (16), we obtain the acoustic frequency spectrum $P_{R}(x, \omega)$ in the reduced form: 


$$
\begin{aligned}
& P_{R}(\boldsymbol{x}, \omega) \\
& \quad=\frac{A N \pi \mu^{2} R^{6} k_{0}^{2}}{4|\boldsymbol{x}|^{2}} \int_{-\infty}^{\infty} \frac{(\boldsymbol{\kappa} \cdot \tilde{\boldsymbol{n}})^{2}|\gamma(\kappa)|^{2} P_{s}(\kappa, \omega) \Psi(\kappa) e^{-2 \kappa R} \mathrm{~d}^{2} \kappa}{\kappa^{2}}
\end{aligned}
$$

\section{Wall-Pressure Wave-Number-Frequency Spectrum}

In Eq. (22) the wall pressure spectrum $P_{s}(\kappa, \omega)$, which would be induced on a fictitious smooth wall by the rough-wall turbulence Reynolds stresses, needs to be specified to evaluate $P_{R}(x, \omega)$. This can be achieved by making use of the available models for the pressure spectrum on a smooth wall and scaling the Reynolds stresses through their dependence on $u_{\tau}^{2}$. Graham [6] has drawn a comparison of different models $\frac{\S}{-}$ for the smooth-wall wave-numberfrequency spectrum of turbulent boundary-layer pressures. In his paper the dimensionless wave-number-frequency spectrum $\tilde{\Phi}_{p}(\kappa, \omega)$ was defined on $x_{2}=0$ in the form

$$
\begin{aligned}
2 \pi \delta\left(\omega-\omega^{\prime}\right) \tilde{\Phi}_{p}\left(\kappa_{1}, \kappa_{3}, \omega\right) \\
\quad=\frac{\omega^{2}}{U_{c}^{2} \Phi(\omega)} \int_{-\infty}^{\infty}\left\langle p(\boldsymbol{x}+\boldsymbol{\Delta}, \omega) p^{*}\left(\boldsymbol{x}, \omega^{\prime}\right)\right\rangle e^{-i \kappa \cdot \boldsymbol{\Delta}} \mathrm{d} \Delta_{1} \mathrm{~d} \Delta_{3}
\end{aligned}
$$

where the tilde on $\tilde{\Phi}_{p}$ indicates that it is normalized by $\Phi(\omega) U_{c}^{2} / \omega^{2}$. The typical eddy convection velocity $U_{c}$ is assumed to be a fixed fraction of the freestream velocity $U$. Both experiments and numerical simulations indicate that $U_{c} \approx 0.5-0.7 U$ with only a weak dependence on the frequency [11]. $\Phi(\omega)$ is the point pressure frequency spectrum whose curve-fitted formulation will be discussed subsequently. Similar to Eq. (20), Eq. (23) may be rearranged by integrating on both sides, yielding

$$
\begin{aligned}
& \tilde{\Phi}_{p}(\boldsymbol{\kappa}, \omega) \\
& \quad=\frac{\omega^{2}}{U_{c}^{2} \Phi(\omega)} \frac{1}{|\gamma(\kappa)|^{2}} \iint_{0}^{\infty} S\left(y_{2}, y_{2}^{\prime} ; \kappa, \omega\right) e^{i\left(\gamma y_{2}-\gamma^{*} y_{2}^{\prime}\right)} \mathrm{d} y_{2} \mathrm{~d} y_{2}^{\prime}
\end{aligned}
$$

For moderately rough surfaces, we assume that the principal features of the wall pressure spectrum would not differ substantially from those of the pressure spectrum on a smooth wall, especially in the vicinity of the convective peak [12]. In this case, by comparing the definitions $(\underline{21})$ and $(\underline{23})$ we can directly relate $\tilde{\Phi}_{p}(\kappa, \omega)$ with $P_{s}(\boldsymbol{\kappa}, \omega)$,

$$
P_{s}(\kappa, \omega)=\frac{U_{c}^{2}}{\omega^{2}} \Phi(\omega) \tilde{\Phi}_{p}(\kappa, \omega)
$$

provided that the principal contributions to the integral in Eq. (24) are from the region $y_{2}, y_{2}^{\prime}>R$. Therefore by substituting Eq. (25) into Eq. (16) and rearranging, the far-field acoustic pressure frequency spectrum $P_{R}(\boldsymbol{x}, \omega)$ may be expressed in terms of the smooth-wall wave-number-frequency spectrum $\tilde{\Phi}_{p}(\boldsymbol{\kappa}, \omega)$,

$$
\begin{aligned}
& P_{R}(\boldsymbol{x}, \omega) \\
& \quad=\frac{A N \pi \mu^{2} R^{6}}{4|\boldsymbol{x}|^{2}} \frac{U_{c}^{2}}{c^{2}} \Phi(\omega) \int_{-\infty}^{\infty} \frac{(\boldsymbol{\kappa} \cdot \tilde{\boldsymbol{n}})^{2}|\gamma(\kappa)|^{2} \tilde{\Phi}_{p}(\kappa, \omega) \Psi(\kappa) e^{-2 \kappa R} \mathrm{~d}^{2} \boldsymbol{\kappa}}{\kappa^{2}}
\end{aligned}
$$

Note that it is now essential to increase $u_{\tau}$ and $\delta$ in the definition of $\tilde{\Phi}_{p}(\kappa, \omega)$ to the rough-wall values to compensate for the enhanced surface drag and turbulence production $[\underline{3}, \underline{20}]$.

${ }^{\S}$ These models are the Corcos model [7], the Efimtsov model [8], the Smol'yakov and Tkachenko model [9], the Ffowcs Williams model [10], the Chase 1 model [11], and the Chase 2 model [12], respectively (see the Appendix for details).

\section{E. Determinations of $u_{\tau}$ and $\delta$}

The evaluation of $\tilde{\Phi}_{p}(\boldsymbol{\kappa}, \omega)$ in Eq. (26) depends on two boundarylayer parameters: 1 ) the friction velocity $u_{\tau}$ and 2) the boundary-layer thickness $\delta$, both of which are enhanced by surface roughness. The principal effect of a rough surface is to alter the structure of the boundary layer near the wall, thereby increasing the surface skin friction. Hence it is necessary to determine the skin friction on a rough wall.

For fully developed roughness flows (i.e., $R e_{\tau}>70$ ) over a flat plate, the effective equivalent height of the roughness particles $k_{s}$ is very much larger than the thickness of the viscous sublayer. In this case the overall skin friction is independent of $R e_{L}$ and is a function of $L / k_{s}$ only [21]. Prandtl and Schlichting [22] derived an interpolation formula for skin friction based on the sand grain roughness experiments of Nikuradse [23]. Mills and Hang [16] compared this formulation with some experimental data sets for fully rough flows over sand grain roughness and found that it gave an average error of $17.5 \%$. They attributed the discrepancy to the failure of the formulation to account for the wake component of the mean velocity profile, and proposed a skin friction formula in the form

$$
\left\{\begin{array}{l}
c_{f}=\left[3.476+0.707 \ln \left(x_{1} / k_{s}\right)\right]^{-2.46} \\
C_{F}=\left[2.635+0.618 \ln \left(L / k_{s}\right)\right]^{-2.57}
\end{array}\right.
$$

where $c_{f}$ and $C_{F}$ denote the curve-fitted local and overall skin friction coefficients, respectively. This formula gives an average error of $2.7 \%$ only when compared to the same data and is valid over a wide parameter range. Therefore the friction velocity $u_{\tau}$ can be obtained through the definition,

$$
u_{\tau}=U \sqrt{c_{f} / 2}
$$

Building on the work of von Kármán [24], Krogstad et al. [25] suggested a relationship to match the mean velocity profile in the logarithmic and outer regions of a turbulent boundary layer over a rough wall,

$$
\frac{u}{u_{\tau}}=\frac{1}{\kappa_{0}} \ln \left(\frac{y}{k_{s}}\right)+B+\frac{2 \Pi_{0}}{\kappa_{0}} w\left(\frac{y}{\delta}\right)
$$

where $\kappa_{0} \approx 0.41$ and $B=8.5$ are empirical constants. The wake function $w(y / \delta)$ is expressed as follows:

$$
w\left(\frac{y}{\delta}\right)=\frac{1}{2 \Pi_{0}}\left[\left(1+6 \Pi_{0}\right)-\left(1+4 \Pi_{0}\right)\left(\frac{y}{\delta}\right)\right]\left(\frac{y}{\delta}\right)^{2}
$$

where $\Pi_{0}$ represents the strength of the wake. The value of $\Pi_{0}$ for a rough surface is typically larger than for a smooth surface, and it varies with different types of surface roughness. In the present study, $\Pi_{0} \approx 0.45$ has been tentatively applied to the rough surface formed by hemispherical bosses because this value gives the best fit to the boundary-layer thickness measured by hot wire (see Fig. 3 ).

The boundary-layer thickness $\delta$ on a rough wall is thus solved from Eqs. (27-30). If we take $y=\delta$ in Eq. (29), then at this location $u \approx U$ and $\overline{w(1)} \equiv 1$. In this case Eq. (29) is simplified as

$$
\frac{U}{u_{\tau}}=\frac{1}{\kappa_{0}} \ln \left(\frac{\delta}{k_{s}}\right)+B+\frac{2 \Pi_{0}}{\kappa_{0}}
$$

The only unknown in Eq. (31) is $\delta$ and it can also be expressed in terms of the skin friction coefficient $c_{f}$,

$$
\delta=k_{s} \exp \left(\kappa_{0} \sqrt{2 / c_{f}}-\kappa_{0} B-2 \Pi_{0}\right)
$$

This expression gives the local boundary-layer thickness developing on a rough plate. The overall value of $\delta$ for the whole plate $\bar{\delta}$ can be obtained by substituting $C_{F}$ for $c_{f}$ in Eq. (32).

Figure $\underline{3}$ shows the predicted local boundary-layer thickness $\delta$ based on Eq. (32) for two types of roughness elements, that is, $R=4 \mathrm{~mm}$ and $\bar{R}=3 \mathrm{~mm}$, respectively. The experimental results 


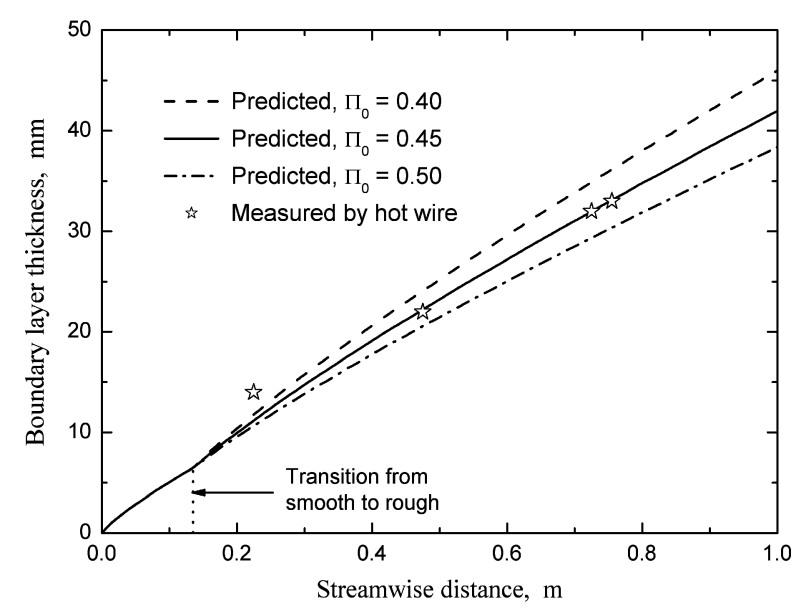

a)

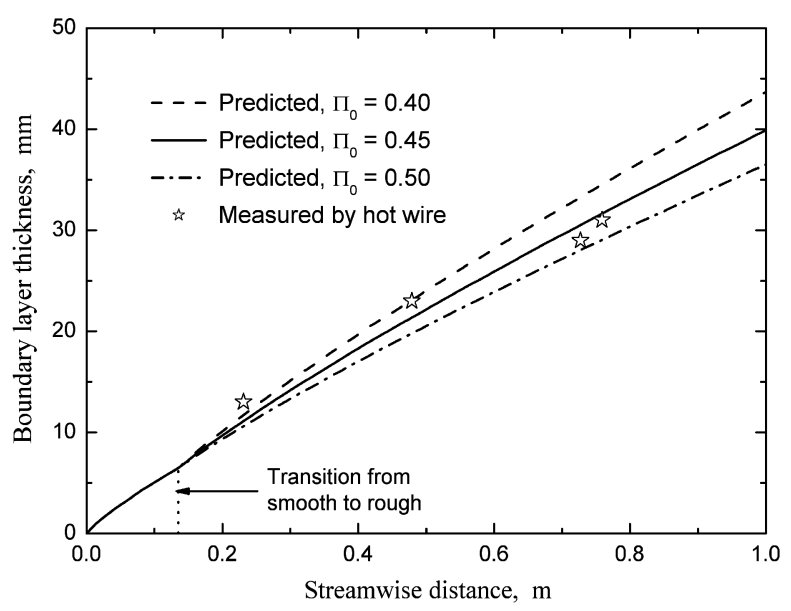

b)

Fig. 3 Comparison between the predicted and measured boundary-layer thickness $\delta$ on a rough plate. a) $R=4 \mathrm{~mm}, \mathrm{~b}) R=3 \mathrm{~mm} ; U=20 \mathrm{~m} / \mathrm{s}$.

through hot-wire measurements at several stations along the flat plate are also shown for comparison. As can be seen in Fig. 3, Eq. (32) with $\Pi_{0}=0.45$ is able to give very close predictions of the rough-wall boundary-layer thickness to the measured values of $\delta$, especially at downstream stations. Noticeable errors occur at upstream stations, but the mean error should be small enough to give an approximate prediction for the overall boundary-layer thickness $\bar{\delta}$.

\section{Evaluation of the Rough-Wall Acoustic Pressure Spectrum}

\section{A. Asymptotic Approximation and Empirical Model}

Howe $[\underline{4}, \underline{13}]$ evaluated the integral in Eq. (26) approximately to obtain an estimate of the radiated roughness noise in the far field. As mentioned before, the principal components of the boundary-layer Reynolds stress occur in the hydrodynamic domain wherein $\kappa=|\kappa| \sim|\omega| / U_{c}$. The wall pressure wave-number-frequency spectrum $\tilde{\Phi}_{p}(\kappa, \omega)$ is expected to be sharply peaked in the neighborhood of a "convective ridge" centered on

$$
\kappa_{1} \sim \omega / U_{c}, \quad \kappa_{3}=0
$$

and the principal contribution to the integral will thus be from the vicinity of the convective peak. The way Howe evaluated the integral in Eq. (26) was to expand the remaining terms in the integrand about the convective peak and integrate term by term. It is essentially a representation of asymptotic approximation, and may be unable to evaluate the integral with sufficient accuracy.

Howe [14] also proposed an empirical model for the rough-wall acoustic frequency spectrum aimed at larger values of $R$ where there may be interstitial flows and wake formation. He assumes this empirical model to be in the form

$$
\frac{\Phi_{R}(\boldsymbol{x}, \omega)}{\rho_{0}^{2} u_{\tau}^{3} \delta} \approx \tau_{0} \frac{A \cos ^{2} \theta}{|\boldsymbol{x}|^{2}} \frac{R}{\delta} \frac{u_{\tau}^{2}}{c_{0}^{2}} \frac{\left(\omega R / u_{\tau}\right)^{3}}{\left[1+\beta\left(\omega R / u_{\tau}\right)^{2}\right]^{n / 2}}
$$

where the empirical coefficients, $\tau_{0}, \beta$, and $n$, are partially estimated by the experimental data of Hersh [15] on sound radiation by sandroughened pipes of various grit sizes. The best fit to Hersh's data can be achieved by taking

$$
\beta=0.0025, \quad n=11
$$

Following Howe [14], the "roughness parameter" $\tau_{0}$ depends primarily on the spacing of the roughness elements, determined by $\sigma$ :

$$
\tau_{0} \approx\left(u_{\tau} / U_{c}\right)^{2}(\sigma / \pi) \quad \text { when } \sigma \ll 1
$$

However, these values of adjustable coefficients should be regarded as tentative because no directivity information is available from the Hersh data. For this reason, although $\beta$ and $n$ are capable of fixing the shape of the spectrum (see Fig. 4), it is not possible to derive the absolute levels from Hersh's data.

\section{B. Direct Numerical Integration}

The direct numerical integration is therefore considered instead to evaluate the integral in Eq. (26). It is convenient to introduce the polar representation $(\kappa, \alpha)$ in the wave number plane, so that

$$
\kappa_{1}=\kappa \cos \alpha, \quad \kappa_{3}=\kappa \sin \alpha
$$

Combining Eq. (37) with the definition of the unit vector in the far field

$$
\tilde{\boldsymbol{n}}=\boldsymbol{x} /|\boldsymbol{x}|=(\cos \theta, \sin \theta \cos \phi, \sin \theta \sin \phi)
$$

yields $\boldsymbol{\kappa} \cdot \tilde{\boldsymbol{n}}$ in the polar coordinates,

$$
\boldsymbol{\kappa} \cdot \tilde{\boldsymbol{n}}=\kappa(\cos \alpha \cos \theta+\sin \alpha \sin \theta \sin \phi)
$$

Therefore the acoustic pressure frequency spectrum $P_{R}(\boldsymbol{x}, \omega)$ becomes

$$
P_{R}(\boldsymbol{x}, \omega)=\frac{A \sigma \mu^{2}}{4|\boldsymbol{x}|^{2}} \frac{R^{4}}{\delta^{* 4}} \frac{U_{c}^{2}}{c^{2}} \Phi(\omega) D(\theta, \phi)
$$

where the nondimensional term $D(\theta, \phi)$ gives the directivity information:

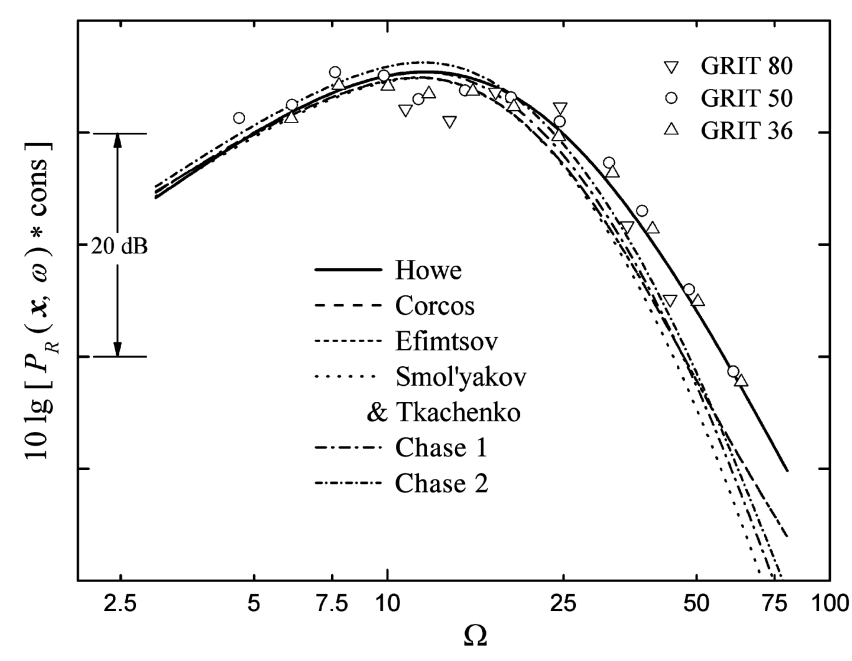

Fig. 4 Roughness noise spectra predicted by the empirical model and numerical integration together with the experimental data by Hersh [15]. 


$$
D(\theta, \phi)=I_{1} \cos ^{2} \theta+I_{2} \sin ^{2} \theta \sin ^{2} \phi+2 I_{3} \cos \theta \sin \theta \sin \phi
$$

In the preceding expression, $I_{1}-I_{3}$ are integrals with respect to the nondimensional polar coordinates $\kappa \delta^{*}$ and $\alpha$,

$$
\begin{gathered}
I_{1}=\int_{0}^{\infty} \int_{0}^{2 \pi} \chi \cos ^{2} \alpha \mathrm{d} \kappa \delta^{*} \mathrm{~d} \alpha \\
I_{2}=\int_{0}^{\infty} \int_{0}^{2 \pi} \chi \sin ^{2} \alpha \mathrm{d} \kappa \delta^{*} \mathrm{~d} \alpha \\
I_{3}=\int_{0}^{\infty} \int_{0}^{2 \pi} \chi \cos \alpha \sin \alpha \mathrm{d} \kappa \delta^{*} \mathrm{~d} \alpha
\end{gathered}
$$

where $\chi$ is a nondimensionalized term,

$$
\chi=\left[|\gamma(\kappa)| \delta^{*}\right]^{2} \tilde{\Phi}_{p}(\kappa, \omega) \Psi(\kappa) e^{-2 \kappa R} \kappa \delta^{*}
$$

It should be noted that the original infinite double integral over rectangular coordinates in Eq. (26) is now reduced to a series of mixed double integral over polar coordinates with directivity information excluded from the integrals, which greatly improves the computation efficiency. The numerical integrations of $I_{1}-I_{3}$ are then carried out by using the five-point Gaussian quadrature with adjustable integration subintervals. Moreover, the infinite $\kappa \delta^{*}$ integral can be reduced to a finite integral with sufficient accuracy thanks to the existence of a weighting function $e^{-2 \kappa R}$ in $\chi$. Hence the infinite upper bound in the $\kappa \delta^{*}$-integral may be substituted by a positive real number, $\kappa \delta^{*} \leq 25 \delta^{*} / R$, to make the numerical integration practical.

It remains to find a suitable form of the point pressure frequency spectrum $\Phi(\omega)$ in Eq. (40). Ahn [26] has approximated a curve fit for the frequency spectrum data in Blake [17],

$$
\begin{aligned}
& \Phi(\omega)=\left(\frac{\tau_{w}^{2} \delta^{*}}{U}\right) \frac{2 \pi 8.28 S h^{* 0.8}}{\left[1+4.1 S h^{* 1.7}+4.4 \times 10^{-4} S h^{* 5.9}\right]} \\
& =\left(\frac{\tau_{w}^{2} \delta^{*}}{U}\right) g\left(S h^{*}\right)
\end{aligned}
$$

It may be assumed that $\delta^{*} \approx \delta / 8$ for practical purposes [14]. In fact there are a number of forms of $\Phi(\omega)$, for example, the Efimsov curve fit [8]. The reason for choosing the form by Ahn is because it is capable of reproducing the features in three different frequency regions as Blake suggested [20], whereas the Efimsov curve fit fails to give these features notably at low and high frequencies.

The main parametric dependency of roughness noise can then be discovered from Eqs. (40-44). By subtituting Eq. (44) into Eq. (40) and integrating $P_{R}(x, \bar{\omega})$ with respect to $\omega$, we obtain the mean square pressure in the form

$$
\left\langle p^{2}(\boldsymbol{x}, t)\right\rangle=\frac{A \sigma \mu^{2} \rho_{0}^{2}}{8 \pi|\boldsymbol{x}|^{2} c^{2}}\left(\frac{R}{\delta^{*}}\right)^{4}\left(\frac{U_{c}}{u_{\tau}}\right)^{2} u_{\tau}^{6} D(\theta, \phi) \int_{-\infty}^{\infty} g\left(S h^{*}\right) \mathrm{d} S h^{*}
$$

This indicates that $\left\langle p^{2}(\boldsymbol{x}, t)\right\rangle$ scales on the sixth power of Mach number, that is, $\left\langle p^{2}(\boldsymbol{x}, t)\right\rangle \sim M^{6}$, suggesting that surface roughness generated noise is a distribution of dipole sources in nature. In addition the mean square pressure is proportional to the rough area $A$ and there is an explicit dependence of $\left\langle p^{2}(\boldsymbol{x}, t)\right\rangle$ on $R^{4}$. However, $R$ also influences the turbulent boundary properties $\delta^{*}$ and $u_{\tau}$ and the wave number contribution to $D(\theta, \phi)$ through the weighting function $e^{-2 \kappa R}$ in the integrals $I_{1}-I_{3}$. The actual dependence on $R$ is therefore more complicated and a full parametric study is carried out in Sec. $\underline{\mathrm{V}}$. C.

\section{Comparison of Empirical Model with Numerical Method}

A comparison between the empirical model of Eq. (34) and the results of the numerical integration of Eqs. (40-45), which will be referred to as the "numerical method," is illustrated in Fig. 4 for the following nondimensional parameter values:

$$
\begin{gathered}
\sigma=0.2, \quad R / \delta=0.01, \quad U_{c} / U=0.6 \\
u_{\tau} / U=0.05, \quad M=0.005
\end{gathered}
$$

These values have been selected to enable the comparison with Howe's empirical model $\Phi_{R}(\boldsymbol{x}, \omega)$, and might be encountered in underwater applications. In these plots the far-field observer is chosen as on the positive $x_{1}$ axis, that is, $\theta=0$ and $\phi=0$, leading $D(\theta, \phi)$ reduced to $I_{1}$.

The numerical integration of $P_{R}(x, \omega)$ is repeated with different wall pressure spectrum models by Corcos [7], Efimtsov [8], Smol'yakov and Tkachenko [9], and Chase [11,12]. The Ffowcs Williams model [10] is rejected, as suggested by Graham [6], because its divergent behavior in high wave number region does not satisfy the integral requirement:

$$
\frac{1}{(2 \pi)^{2}} \iint_{-\infty}^{\infty} \tilde{\Phi}_{p}\left(\kappa_{1}, \kappa_{3}, \omega\right)\left(\frac{U_{c}}{\omega}\right)^{2} \mathrm{~d} \kappa_{1} \mathrm{~d} \kappa_{3}=1
$$

The curves in Fig. 4 represent the variations of $\Phi_{R}(\boldsymbol{x}, \omega)$ and $P_{R}(\boldsymbol{x}, \omega)$ as functions of the dimensionless frequency $\Omega=\omega R / u_{\tau}$. Hersh's data [15] for sand-roughened pipes of various grit sizes are also shown as scatter points. No comparison of the absolute levels of the predicted and measured noise is possible due to the unknown effects of acoustic refraction by the freejet shear layers downstream of the nozzle exit [13]. Accordingly, the heights of the curves have been adjusted to yield the best fit to the data points.

As shown in Fig. 4, the shape of the empirical spectrum $\Phi_{R}(\boldsymbol{x}, \omega)$ fits the experimental data very well by taking the coefficient values in Eq. (35). Moreover, the comparison between the empirically and numerically predicted roughness noise spectra exhibits encouraging agreement. Both $\Phi_{R}(\boldsymbol{x}, \omega)$ and $P_{R}(\boldsymbol{x}, \omega)$ peak around $\Omega \approx 13$ and decay rapidly at higher frequencies. The relatively abrupt falloff of $P_{R}(\boldsymbol{x}, \omega)$ is due to the features of $\Phi(\omega)$ specified in Eq. (44). In addition, the numerically predicted spectra obtained from different models of $\tilde{\Phi}_{p}(\boldsymbol{\kappa}, \omega)$ agree with one another very well. In this dimensionless frequency range $(3 \leq \Omega \leq 80)$, the Efimtsov model reduces to the Corcos model and thus their curves coincide, predicting levels comparable to other models except when $\Omega>60$.

\section{Experimental Validation}

\section{A. Experimental Setup}

The experiments were performed in an open jet of a low-speed wind tunnel to measure the radiated sound from a rough plate. A schematic of the experimental setup is shown in Fig. 5. The wind tunnel has a inner cross section of $0.586 \times 0.35 \mathrm{~m}$ and a velocity range of $0-31.0 \mathrm{~m} / \mathrm{s}$; it was lined with plastic foam on the inner wall to reduce the fan noise and flow noise traveling inside the tunnel. A flat plate was placed in the center of the open jet with the plate plane perpendicular to the ground. The plate surface was partially roughened in a $0.64 \times 0.64 \mathrm{~m}$ square region by rigid hemispherical plastic beads placed in parallel rows. Each row spans the entire height of the rough region. This was achieved by drilling a number of hemispherical holes into four modeling boards with spherical beads glued into these holes, and then mounting these boards flush in a square recess milled into the flat plate. Two different surface roughness conditions were examined: 1) $R=4 \mathrm{~mm}, \sigma=0.50$ and 2) $R=3 \mathrm{~mm}, \sigma=0.44$; together with a smooth surface for comparison. The values of both $R$ and $\sigma$ were chosen comparatively high to ensure that the roughness noise could be detected by the microphones.

The leading edge of the flat plate was carefully streamlined to reduce sound scattering effects due to flow separation at a bluffheaded nose. The boundary layer was tripped using four layers of tape cut in sawtooth form with $0.13 \mathrm{~mm}$ in thickness and $1.5 \mathrm{~mm}$ in 


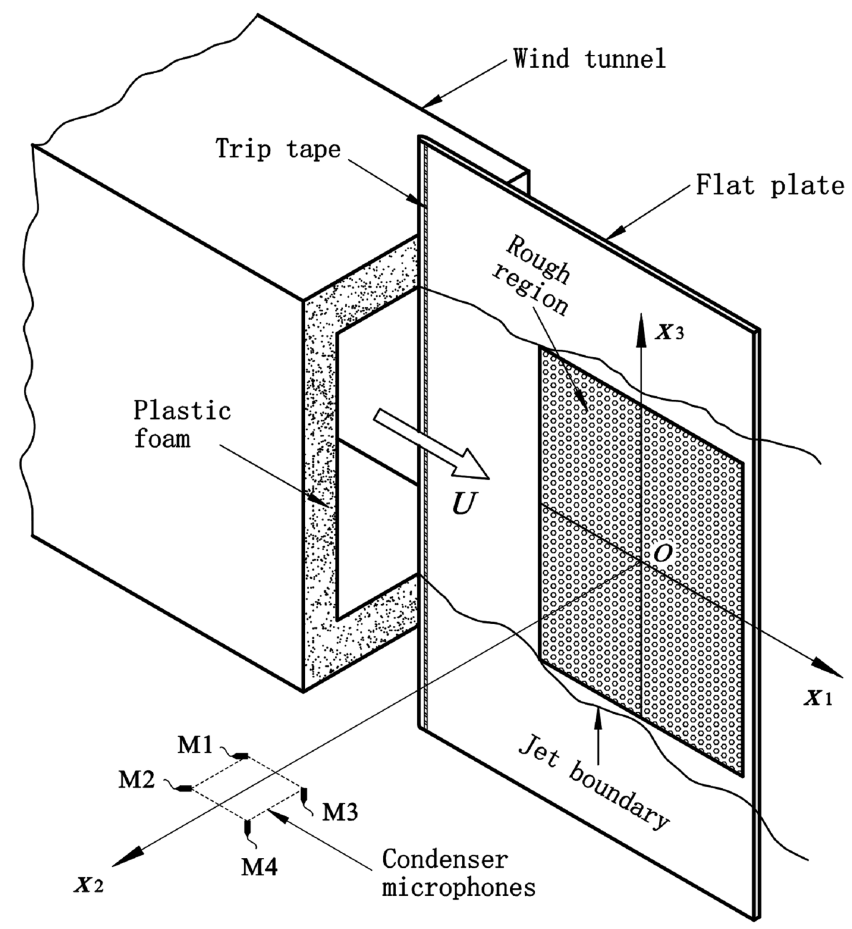

Fig. 5 Schematic of the experimental setup.

width. The tape was placed $4 \mathrm{~cm}$ from the leading edge over the entire span of the plate. This encourages a fully developed turbulent boundary layer close to the leading edge. Also, the rough region was located at $0.34 \mathrm{~m}$ from the leading edge of the plate to satisfy the assumption that the roughness elements are contained entirely within the boundary layer.

In the acoustic measurements the radiated sound from the rough plate was detected by four $1 / 2$-in. free-field condenser microphones of Brüel and Kjær (B\&K) as marked in Fig. 5. The acoustic signals were acquired for a duration of $60 \mathrm{~s}$ at a sampling frequency of $65.5 \mathrm{kHz}$ in the frequency range $7 \mathrm{~Hz}-25.6 \mathrm{kHz}$. The Cartesian coordinates are also indicated in the schematic with the origin at the center of the rough region. The four $\mathrm{B} \& \mathrm{~K}$ microphones were arranged in a $0.16 \times 0.16 \mathrm{~m}$ square array on the plane $x_{3}=0$ and axisymmetric to the plane $x_{1}=0$ with microphones M1 and M2 upstream and M3 and M4 downstream. To reduce unwanted turbulence fluctuations around the microphones (wind noise), it is important to put the microphones out of the jet boundary, and hence the distance between the plate plane and front microphones M1 and M3 was set as $0.6 \mathrm{~m}$.

\section{B. Modifications of the Numerical Method}

For the experimental setup illustrated in Fig. 5, the numerical method (40) should be accordingly modified to enable the comparison of the numerical prediction with experimental results.

Firstly, the nonuniform directivity function $D(\theta, \phi)$ for sound radiated from different parts of the rough region should be considered as the far-field distance $|\boldsymbol{x}|$ and the dimensions of the rough area $A$ are now comparable. In this case the rough area $A$ should be divided into a number of equal subareas $A_{j}, j=1, \ldots, N_{A}$; then one can apply the numerical integration to determine the roughness noise radiated from each subarea, and add them up to obtain the total radiation from the whole rough region. For the present experimental setup, $|\boldsymbol{x}| \gg \sqrt{A_{j}}$ can be satisfied if the number of subareas $N_{A}>12 \times 10$ is taken. The growing boundary-layer thickness along the plate chord is also taken into account.

Secondly, the cross spectra were used for the measured noise spectra to discard the interference of uncorrelated noise signals. The microphones were located outside the flow region, but some unwanted noise (e.g., noise from the air supply) still exists as the measurements were not performed in an anechoic chamber. The cross-correlation technique was therefore applied to pick out radiated sound from the rough plates. Accordingly the numerical prediction should also consider the cross correlation between two close microphones. We assume that the noise signals obtained by the two microphones, that is, $p\left(\boldsymbol{x}_{1}, t_{1}\right)$ and $p\left(\boldsymbol{x}_{2}, t_{2}\right)$, are well correlated, so that

$$
p\left(x_{2}, t_{2}\right)\left|x_{2}\right|=p\left(x_{1}, t_{1}+\Delta t\right)\left|x_{1}\right|
$$

where $\Delta t$ is the difference in observer time of two microphones,

$$
\Delta t=t_{2}-t_{1}=\left(\left|x_{2}\right|-\left|x_{1}\right|\right) / c
$$

Hence the Fourier transform of $p\left(\boldsymbol{x}_{1}, t_{1}\right)$ and $p\left(\boldsymbol{x}_{2}, t_{2}\right)$ can be related,

$$
\hat{p}\left(\boldsymbol{x}_{2}, \omega^{\prime}\right)=\hat{p}\left(\boldsymbol{x}_{1}, \omega\right) \frac{\left|\boldsymbol{x}_{1}\right|}{\left|\boldsymbol{x}_{2}\right|} e^{-i \omega \Delta t}
$$

and the power spectral density becomes

$$
\left\langle\hat{p}\left(x_{1}, \omega\right) \hat{p}^{*}\left(x_{2}, \omega^{\prime}\right)\right\rangle=\left|\hat{p}\left(x_{1}, \omega\right)\right|^{2} \frac{\left|x_{1}\right|}{\left|x_{2}\right|} e^{i \omega \Delta t}
$$

where $e^{i \omega \Delta t}$ is an extra phase term. Similarly, the cross spectrum of predicted roughness noise for two microphones can be approximated by taking the average far-field distance $|\overline{\boldsymbol{x}}|=\sqrt{\left|\boldsymbol{x}_{1}\right|\left|\boldsymbol{x}_{2}\right|}$ and average directivity angles $(\bar{\theta}, \bar{\phi})$ in Eq. (40).

Taking into these two factors, the numerical method (40) becomes

$$
P_{R}(\overline{\boldsymbol{x}}, \omega)=\sum_{j=1}^{N_{A}} \frac{A_{j} \sigma \mu^{2}}{4|\overline{\boldsymbol{x}}|^{2}} \frac{R^{4}}{\delta_{j}^{* 4}} \frac{U_{c}^{2}}{c^{2}} \Phi(\omega) D\left(\bar{\theta}_{j}, \bar{\phi}_{j}\right) e^{i \omega \Delta t}
$$

which expresses the cross spectrum of roughness noise radiated from a total of $N_{A}$ subareas.

It should also be noted that in Fig. $\underline{5}$ the rough region starts at $0.34 \mathrm{~m}$ from the leading edge, whereas it is assumed in the skin friction formula (27) that the surface is roughened on the whole plate. This contradiction can be removed by a correction to the length of the rough region. As shown in Fig. 6, the boundary-layer thickness after the trip tape can be approximated as the turbulent boundary layer starts from the leading edge. This boundary layer grows along the front smooth surface until it achieves a thickness $\delta_{0}$ at the edge of the rough region. $\delta_{0}$ can be calculated by means of the $1 / 9$ power law for smooth walls [27],

$$
\delta_{0}=0.36 x_{\mathrm{le}} / R e_{\mathrm{le}}^{1 / 6}
$$

In another way $\delta_{0}$ can be assumed as produced by a hypothetical extension $x_{0}$ of the rough region. Substituting this value of $\delta_{0}$ into

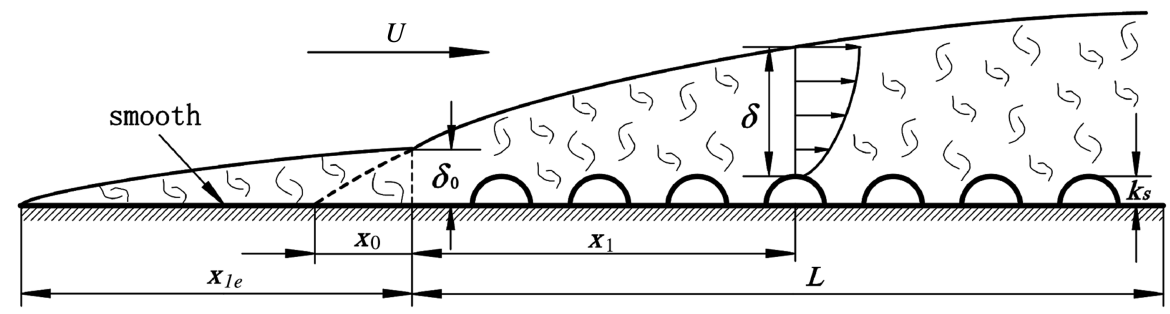

Fig. 6 Schematic of the rough region located at a distance from the leading edge. 


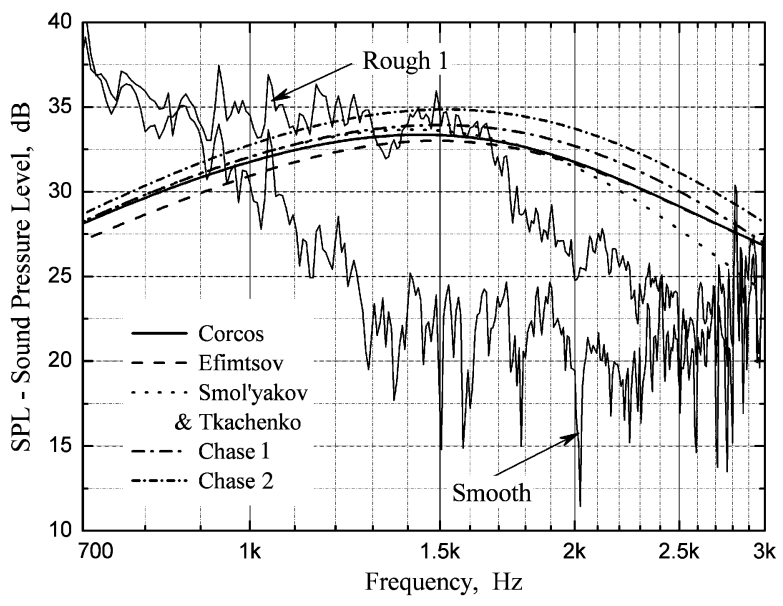

a) Rough $1, R=4 \mathrm{~mm}, \sigma=\mathbf{0 . 5 0}$

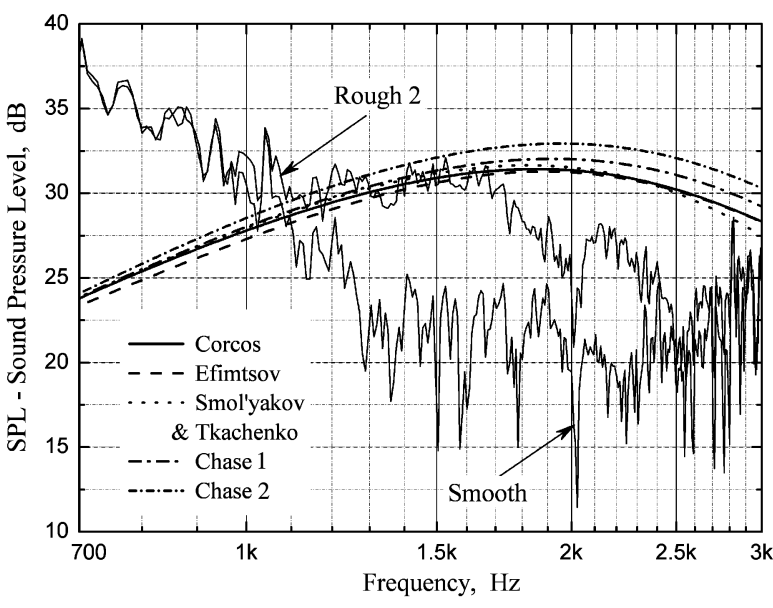

b) Rough $2, R=3 \mathrm{~mm}, \sigma=0.44$

Fig. 7 Comparison between experimental and predicted roughness noise spectra. Smooth-wall experimental results are shown for comparison.

Eq. (32) and combining Eq. (27) gives the correction term

$$
x_{0}=k_{s} \exp \left[\left(c_{f 0}^{-1 / 2.46}-3.476\right) / 0.707\right]
$$

where $c_{f 0}$ is the local skin friction coefficient at $x_{\text {le }}$,

$$
c_{f 0}=2 /\left[\ln \left(\delta_{0} / k_{s}\right) / \kappa_{0}+B+2 \Pi_{0} / \kappa_{0}\right]^{2}
$$

Therefore the length scales in the skin friction formula (27), that is, $x_{1}$ and $L$, are corrected to $x_{1}+x_{0}$ and $L+x_{0}$, respectively, for the rough region not starting from the leading edge.

\section{Results and Discussion}

The sound pressure spectra for the rough and smooth plates measured by the B\&K microphones are shown in Fig. 7 and compared with the numerically predicted roughness noise spectra using different wave-number-frequency spectrum models. The acoustic measurements were performed for a series of flow velocities, $U=10,15, \ldots, 30 \mathrm{~m} / \mathrm{s}$, but only the experimental results for $U=30 \mathrm{~m} / \mathrm{s}$ are presented because the surface roughness generated noise is too quiet at lower flow velocities to reveal evident differences between the noise spectra of rough and smooth plates. The roughness noise scales as $U^{6}$ and so is more evident above the TE noise which scales as $U^{5}$ at the higher velocity. The cross-spectra results are presented for the microphone pair, M3 and M4, because it showed the greatest signal to noise. The experimental data were processed in narrow band and divided by the bandwidth $\Delta f=8 \mathrm{~Hz}$.

As shown in Fig. 7, the measured noise spectra are significantly contaminated by facility noise. The background noise dominates at most of the frequencies including noticeable peaks at $3 \mathrm{kHz}$ which are from the motor and fan of the wind tunnel. At the low frequencies $(f<800 \mathrm{~Hz})$ the dominating noise is supposed to be from the air supply. The predicted TE noise spectrum peaks around $100 \mathrm{~Hz}$ with very low spectral levels, and there is no difference between the measured background noise spectra with a smooth plate and without any plate. This indicates that the contribution of TE noise to the total noise spectrum is negligible.

However, the measured noise spectra of the rough plates are still above that of the smooth plate between $1-2.5 \mathrm{kHz}$, making the roughness noise detectable from the noise of the smooth plate. This appears to be confirmed by the fact that the rough-plate spectra are less fluctuating than that of the smooth plate in this frequency range, which suggests that they contain statistically steady sources actively radiating sound. Comparing results for the two roughness elements, one notices that the sound from the smaller roughness element displays a lower spectral peak, though not very distinct, by about $5 \mathrm{~dB}$ at a slightly higher frequency. Some beamforming source maps obtained through a phased microphone array also confirm that the rough plates have remarkably higher acoustic source strengths than the smooth plate in this frequency range. Moreover, it is shown that different wave-number-frequency spectrum models produce approximately the same roughness noise spectra.

The measured and predicted roughness noise spectra show a reasonable amount of agreement. The numerical method is capable of predicting radiated roughness noise at approximately the same absolute levels as the experimental results in the frequency range 1$1.7 \mathrm{kHz}$, although the difference becomes notable at higher frequencies. One possible reason for the discrepancy in $f>1.7 \mathrm{kHz}$ frequency is that the approximate Green function in Eq. (5) is based on the assumption, $k_{0} \delta \ll 1$. Take Rough 1 for example, the overall boundary-layer thickness over the rough region $\bar{\delta} \sim 2.9 \mathrm{~cm}$, and thus the preceding assumption is not met when $f=c / 2 \pi \bar{\delta} \sim 1.8 \mathrm{kHz}$. Another reason could be inaccuracy in the models of smooth-wall wave-number-frequency spectrum. The adjustable coefficients in these models are fixed by comparison with experimental data. However, there is no completely satisfactory theoretical understanding of the characteristics of the smooth-wall pressure spectrum so far.

\section{Numerical Prediction and Analysis}

\section{A. Roughness Noise from an Aircraft Wing}

The comparisons of both the spectral shape and absolute level of the predicted roughness noise with both the empirical model and experimental results have provided some validation of the diffraction theory of turbulent boundary-layer generated roughness noise. On this basis the diffraction theory and numerical method were employed to investigate the effects of surface roughness on airframe noise. Herein the far-field roughness noise generated by an aircraft wing will be numerically predicted and compared with the corresponding TE noise.

The roughness noise prediction is based on the approximate dimensions of the wing of a Boeing 757 sized aircraft. The wing has been estimated in the model as a flat plate with the following dimensional parameters:

$$
L_{c} \approx 5 \mathrm{~m}, \quad L_{s} \approx 16.5 \mathrm{~m}, \quad A=L_{c} L_{s} \approx 82.5 \mathrm{~m}^{2}
$$

The aircraft noise is most annoying to the residents outside the airport perimeter in a typical built-up area when the airplane is approaching to or taking off from the airport. In this case the typical Mach number $M$ of the freestream velocity and far-field observer may be taken as

$$
M=U / c_{0}=0.2, \quad|x|=500 \mathrm{~m}, \quad \theta=\pi / 4, \quad \phi=0
$$

Note that in this direction the directivity function $D(\theta, \phi)$ in Eq. $(\underline{41})$ is reduced to $I_{1} / 2$.

Furthermore, it is necessary to evaluate the ensemble average $\left\langle p^{2}(\boldsymbol{x}, t)\right\rangle$ of the far-field acoustic pressure by integrating the 
frequency spectrum $P_{R}(x, \omega)$ over the audio frequency range, $f \in[20 \mathrm{~Hz}, 20 \mathrm{kHz}]$. A-weighting [28] is also taken into account in the integration to yield a more accurate noise prediction. Provided that $P_{R}(\boldsymbol{x}, \omega)$ is an even function and $\omega=2 \pi f$, the representation of $\left\langle p^{2}(\boldsymbol{x}, t)\right\rangle$ in Eq. (15) becomes

$$
\left\langle p^{2}(\boldsymbol{x}, t)\right\rangle=\frac{1}{\pi} \int_{40 \pi}^{4 \times 10^{4} \pi} 10^{C_{A} / 10} P_{R}(\boldsymbol{x}, \omega) \mathrm{d} \omega
$$

Exact values of $C_{A}$ are not available for all definite frequencies; hence interpolation is used if necessary.

Finally, the sound pressure level (SPL) of the rough noise spectrum is obtained by comparing $P_{R}(x, \omega)$ with the reference sound pressure level

$$
\mathrm{SPL}=10 \log _{10}\left[\frac{P_{R}(\boldsymbol{x}, \omega)}{4 \times 10^{-10} \mathrm{~Pa}^{2}}\right], \mathrm{dB}
$$

Similarly, the overall sound pressure level (OASPL) is given by

$$
\text { OASPL }=10 \log _{10}\left[\frac{\left\langle p^{2}(\boldsymbol{x}, t)\right\rangle}{4 \times 10^{-10} \mathrm{~Pa}^{2}}\right], \mathrm{dBA},
$$$$
f \in[20 \mathrm{~Hz}, 20 \mathrm{kHz}]
$$

For comparison, the corresponding TE noise was calculated based on the same dimensions of the aircraft wing for both rough and smooth surfaces. The surface roughness increases the friction velocity $u_{\tau}$ and boundary-layer thickness $\delta$, as mentioned before, and hence similar increases will be expected for the TE noise of a rough surface. However, the smooth-wall TE noise was chosen as the comparison reference because the surface roughness was generally not taken into account in the traditional estimate of airframe noise, and hence its effects will be more explicit if the roughness noise is compared with the traditionally predicted TE noise. Moreover, to be consistent with the roughness noise prediction, the TE noise is also predicted on the basis of an empirical model by Howe [14] with Chase's formula [11] of wall pressure spectrum. The directivity function in this model has been taken as 1 to obtain the maximum sound radiation.

\section{B. Idealized Test Cases}

Three idealized test cases were considered to approximately quantify the possible roughness noise from the wing of a Boeing 757 sized aircraft:

Case 1: Rough surfaces formed by rivets, ribs, joints, etc.

Case 2: Rough surfaces formed by environmental contamination

Case 3: Dip-galvanized metal surfaces

The relative parameters of test cases 1-3 are listed in Table 1 with the corresponding values of aerodynamically smooth surfaces for comparison. As shown in Table 1 , cases 1 and 2 can be categorized as fully developed roughness $\left(R e_{\tau}^{-}>70\right)$ and hence the skin friction formula Eq. (27) is applicable, whereas case 3 is in fact transitional roughness $\left(5<R e_{\tau}<70\right)$ in which the overall skin friction coefficient $C_{F}$ is dependent on both $R e_{L}$ and $L / k_{s}$. For convenience Eq. (27) is still applied to case 3 in the calculation, but it should be noted that the actual skin friction is a bit smaller. As previously mentioned, the values of $u_{\tau}$ and $\delta$ for cases 1 and 2 have been increased substantially due to the enhanced surface drag and turbulence production. However, because of the very small roughness height, the surface of case 3 appears more like a smooth wall with similar parameter values.
Note that the roughness elements in these test cases are generally not perfect hemispheres, and $R$ herein corresponds to the equivalent roughness height. The value of $\sigma$ varies with the different rough surfaces. It is reasonable to take $\sigma$ as small as 0.05 for an aerofoil surface with a sparse distribution of rivets, ribs, joints, etc., whereas for the other cases the roughness elements are assumed distributed very densely over the aerofoil surface. Following Howe [4], the maximum value of roughness density, $\sigma_{\max } \sim 0.91$, occurs for a hexagonal close packing, but this would not be representative for a purely random distribution. The value of $\sigma$ for a square close packing is $\sigma_{\mathrm{sq}} \sim 0.78$. Hence the mean value of $\sigma_{\max }$ and $\sigma_{\mathrm{sq}}, \sim 0.85$, is tentatively used as the practical maximum $\sigma$ in cases 2 and 3 .

The predicted roughness noise spectra for the three test cases are depicted in Fig. $\underline{8}$, and compared with corresponding TE noise spectra for both rough and smooth surfaces. Similar to Fig. 4, different models of wave-number-frequency spectrum produce approximate roughness noise spectra, especially around the peaks where all these curves almost coincide with one another. As can be seen in Fig. 8, the smooth-wall TE noise spectra are significant only in the low-frequency region. They always peak around very low frequencies, $\sim 65 \mathrm{~Hz}$, then decay monotonically with increasing frequencies. The roughness noise spectra, however, can be nearly ignored in the low-frequency region but achieve their peaks at very high frequencies. This tends to confirm that the roughness noise can be comparable to or even more significant than the corresponding TE noise provided with sufficiently high frequencies.

For case 1, a distinct spectral peak of roughness noise occurs around $2 \mathrm{kHz}$ and is at the approximate level as the peak of the smooth-wall TE noise. However, for cases 2 and 3, the former becomes obviously lower than the latter due to the diminishing size of roughness elements. Nevertheless, the roughness noise spectra of case 2 are still significantly above that of the TE noise spectrum in the frequency range $3-100 \mathrm{kHz}$. In contrast, case 3 shows significantly lower roughness noise spectra with the maximum SPL $<-20 \mathrm{~dB}$. This is because the very small roughness elements in case 3 , that is, $R=0.152 \mathrm{~mm}$, give the dip-galvanized metal surface similar features of a smooth wall. Other possible surface finishes of an aerofoil, for example, camouflage paint in mass production spraying $(R=0.0305 \mathrm{~mm})$, smooth matte paint $(R=0.0064 \mathrm{~mm})[21]$, generate even quieter roughness noise and are therefore not considered further.

Surface roughness also has important effects on the TE noise through enhanced friction velocity $u_{\tau}$ and boundary-layer thickness $\delta$. As observed in Fig. $\underline{\text { a }}$, the rough-wall TE noise is considerably increased for case 1 with a $10.7 \mathrm{~dB}$ higher spectral peak at an even lower frequency, $\sim 35 \mathrm{~Hz}$, than that of the smooth-wall TE noise. However, the effects of surface roughness are not so evident in Figs. $8 \mathrm{~b}$ and $8 \mathrm{c}$. The differences between the rough- and smooth-wall TE noise SPL are only $1.0-3.4 \mathrm{~dB}$ for case 2 and $0.9 \mathrm{~dB}$ at most for case 3 in the whole frequency range, $20 \mathrm{~Hz}-200 \mathrm{kHz}$.

Attention is then confined to the audio frequency range, $f \in[20 \mathrm{~Hz}, 20 \mathrm{kHz}]$. Table 2 presents the predicted roughness noise OASPL in this frequency range and it suggests the same comparative relationship between the roughness noise and TE noise. For case 1, the roughness noise OASPL is higher than the smooth-wall TE noise OASPL by $20 \mathrm{dBA}$ in average; the rough-wall TE noise OASPL is also enhanced by $6 \mathrm{dBA}$. Similarly, the predicted OASPL of case 2 shows a much smaller difference of 3-4 dBA between the roughness noise and smooth-wall TE noise, and the surface roughness increases the TE noise OASPL by only $2 \mathrm{dBA}$. In case 3 , however, the OASPL

Table 1 Test case parameters

\begin{tabular}{lcccccccr}
\hline \hline Case no. & $R, \mathrm{~mm}$ & $\sigma$ & $C_{F}$ & $u_{\tau}, \mathrm{m} / \mathrm{s}$ & $u_{\tau} / U$ & $\bar{\delta}, \mathrm{cm}$ & \multicolumn{1}{c}{$R / \bar{\delta}$} & $R_{\tau}$ \\
\hline 1 & 5.0 & 0.05 & 0.0060 & 3.72 & 0.055 & 11.28 & 0.0443 & 1272.86 \\
2 & 0.5 & 0.85 & 0.0036 & 2.90 & 0.043 & 9.38 & 0.0053 & 99.26 \\
3 & 0.152 & 0.85 & 0.0029 & 2.59 & 0.038 & 8.91 & 0.0017 & 26.98 \\
4a & - & - & 0.0027 & 2.52 & 0.037 & 6.07 & - & - \\
\hline \hline
\end{tabular}

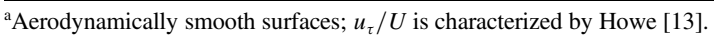




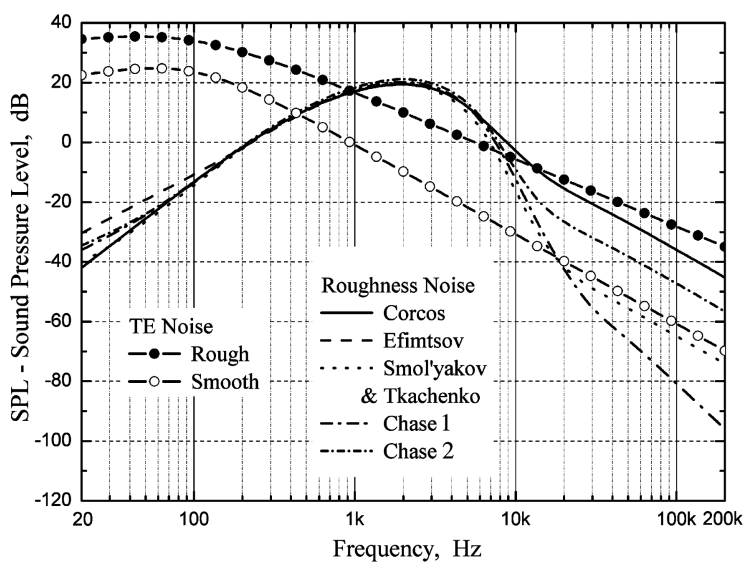

a) Case $1, R=5 \mathrm{~mm}, \sigma=0.05$

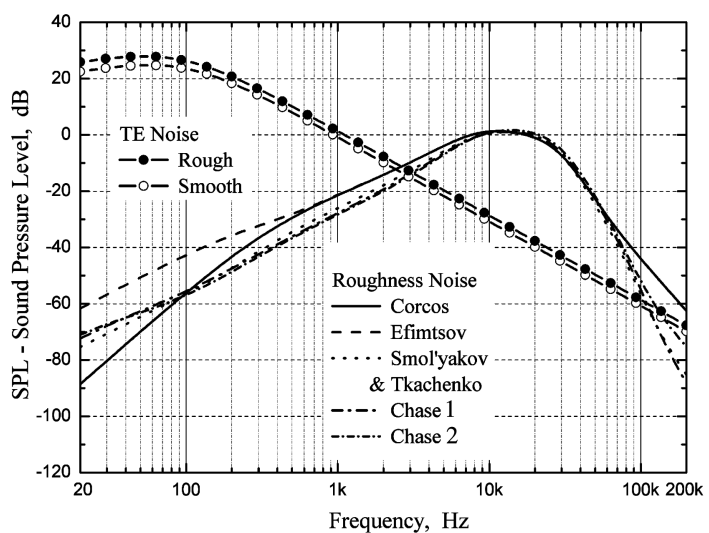

b) Case 2, $R=0.5 \mathrm{~mm}, \sigma=0.85$

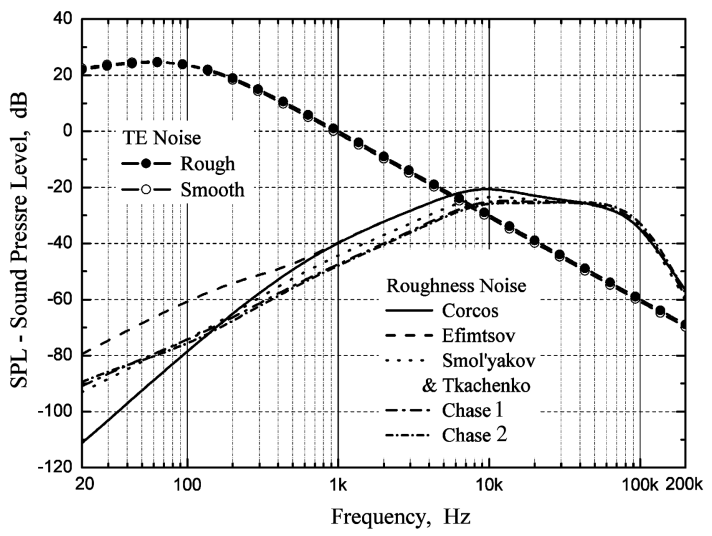

c) Case 3, $R=0.152 \mathrm{~mm}, \sigma=0.85$

Fig. 8 Predicted roughness noise spectra and TE noise spectra for test cases 1-3.

of roughness noise decreases greatly due to the very small roughness height, and becomes 17-23 dBA lower than that of the TE noise. For the same reason the difference between the rough- and smooth-wall TE noise OASPL is merely $1 \mathrm{dBA}$. These features tend to support the

Table 2 Predicted roughness noise and TE noise OASPL for test cases 1-3

\begin{tabular}{lrrrrrrr}
\hline \hline Case no. & \multicolumn{4}{c}{ Roughness noise, $\mathrm{a}$ dBA } & \multicolumn{3}{c}{ TE noise, dBA } \\
\cline { 2 - 8 } & 1 & 2 & 3 & \multicolumn{1}{c}{4} & 5 & Smooth & Rough \\
\cline { 2 - 8 } 1 & 50 & 50 & 49 & 50 & 51 & 30 & 36 \\
2 & 34 & 34 & 33 & 33 & 33 & 30 & 32 \\
3 & 13 & 13 & 10 & 8 & 7 & 30 & 31 \\
\hline \hline
\end{tabular}

ampirical models for smooth-wall pressure spectrum: 1-Corcos, 2-Efimtsov, 3Smol'yakov and Tkachenko, 4-Chase 1,5-Chase 2. speculation that the surface roughness generated noise would contribute substantially to the airframe noise of a clean configuration and the TE noise would be enhanced by the surface roughness to some extent provided that the roughness elements are not too small in size.

\section{Parametric Study}

A parametric study was carried out to investigate the effects of two important parameters, roughness height $R$ and roughness density $\sigma$, on the roughness noise from a Boeing 757 sized aircraft wing.

In Fig. 9a, the roughness noise spectra for different values of $R$ are compared in a wide frequency range, $20 \mathrm{~Hz}-200 \mathrm{kHz}$, with the value of $\sigma$ fixed to 0.85 . As $R$ decreases from $5.0 \mathrm{~mm}$ the spectral peak decreases and shifts to progressively higher frequencies until $R=0.1 \mathrm{~mm}$, where there is no evident peak for $R \leq 0.1 \mathrm{~mm}$. In addition Fig. 9a implies that the peak frequency of the roughness noise spectrum is approximately proportional to $R^{-1}$. Below the peak frequency, the approximate parametric dependency SPL $\sim(\omega R)^{4}$ can be observed.

The dependence of roughness noise OASPL on roughness height $R$ for various values of $\sigma$ is depicted in Fig. 10a. It is shown that the parameter $R$ has significant effects on the roughness noise OASPL. For $R<1 \mathrm{~mm}$, the OASPL varies as $R^{\varepsilon}$, where $3.2<\varepsilon<4.3$ dependent on the value of $\sigma$; whereas for larger values of $R$, the OASPL increases less rapidly with increasing roughness height.

Roughness density $\sigma$ is another important parameter for the roughness noise. The roughness noise spectra for $R=5.0 \mathrm{~mm}$ and different values of $\sigma$ are compared in Fig. $9 \mathrm{~b}$. As $\sigma$ increases from 0.05 the spectral peak shifts to progressively higher frequencies with increasing SPL, though not as obviously as the variation with $R$ in Fig. 9a. This implies that the spectral peak of roughness noise is also related to the distribution of roughness elements. A denser distribution results in the higher peak SPL and peak frequency because there are more roughness elements interacting with turbulence near field and scattering sound.

Figure 10 shows the dependence of roughness noise OASPL on roughness density $\sigma$. As observed in Fig. 10a, larger values of $\sigma$ usually generate louder roughness noise for large roughness elements $(R>2.0 \mathrm{~mm})$. The OASPL variation in Fig. $10 \mathrm{~b}$ also shows that at low values of $\sigma$ the mean square pressure scales approximately on $\sigma$. However, it is not always the case. In Fig. 10b for $R \leq 1.0 \mathrm{~mm}$, the roughness noise OASPL increases slowly with $\sigma$, attains a maximum at $\sigma_{p}$, and then decreases. The peak value $\sigma_{p}$ shifts, as $R$ increases, to a larger value of $\sigma$ with higher OASPL. This is because for larger values of $\sigma$ than $\sigma_{p}$ the wall appears gradually "smoother" as roughness elements become progressively compacter. But this effect will be counteracted to some extent by larger roughness heights $(R \geq 2.0 \mathrm{~mm})$ because the absolute space among the roughness elements increases with $R$ even for a very dense distribution.

Nevertheless, the effect of roughness density $\sigma$ on roughness noise prediction is not as significant as that of roughness height $R$ due to the different OASPL dependencies on $R$ and $\sigma$. For instance, the OASPL variation in Fig. 10b is at most $17.4 \mathrm{dBA}$ from $\sigma=0.01$ to 1.0 for the case $R=5.0 \mathrm{~mm}$, although such a large roughness density of 1.0 is not achievable in practice, which is greatly less than the OASPL increase of at least $40 \mathrm{dBA}(\sigma=0.05)$ from $R=0.1 \mathrm{~mm}$ to $5.0 \mathrm{~mm}$ in Fig. 10a.

\section{Directivity}

In previous roughness noise predictions the far-field observer is fixed at $|x|=500 \mathrm{~m}, \theta=\pi / 4, \phi=0$, and the directivity function $D(\theta, \phi)$ is therefore reduced to $I_{1} / 2$. However, the roughness noise radiation will vary in different directions as described in Eq. (41). The term $I_{1} \cos ^{2} \theta$ describes the effect of a dipole in the flow direction. The contribution of the term $I_{2} \sin ^{2} \theta \sin ^{2} \phi$ to $D(\theta, \phi)$ should be taken

"Hereafter for simplicity the numerical integration is based on the Smol'yakov and Tkachenko model [9] only because all wall pressure models give very similar plots. 


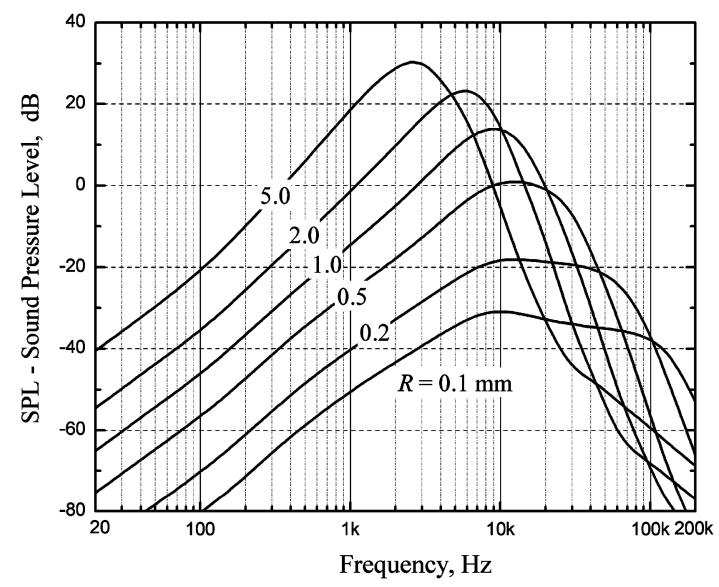

a) Effect of $R, \sigma=0.85$

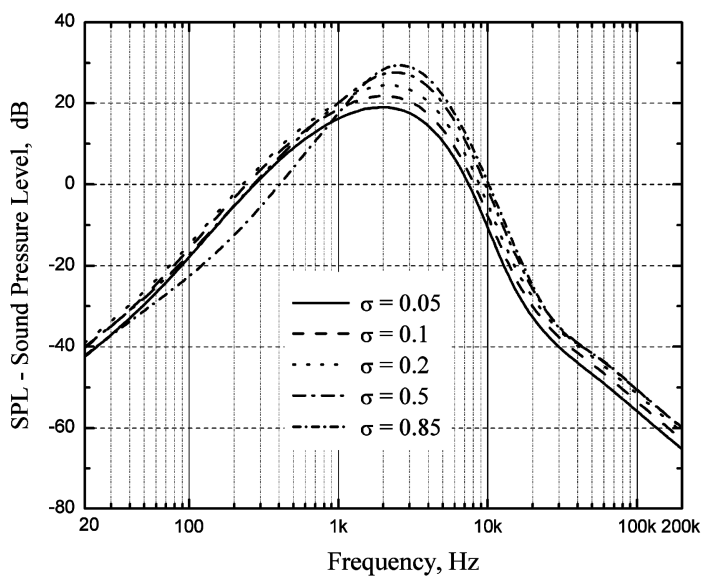

b) Effect of $\sigma, R=5.0 \mathrm{~mm}$

Fig. 9 Effects of roughness height $R$ and roughness density $\sigma$ on predicted roughness noise spectra.

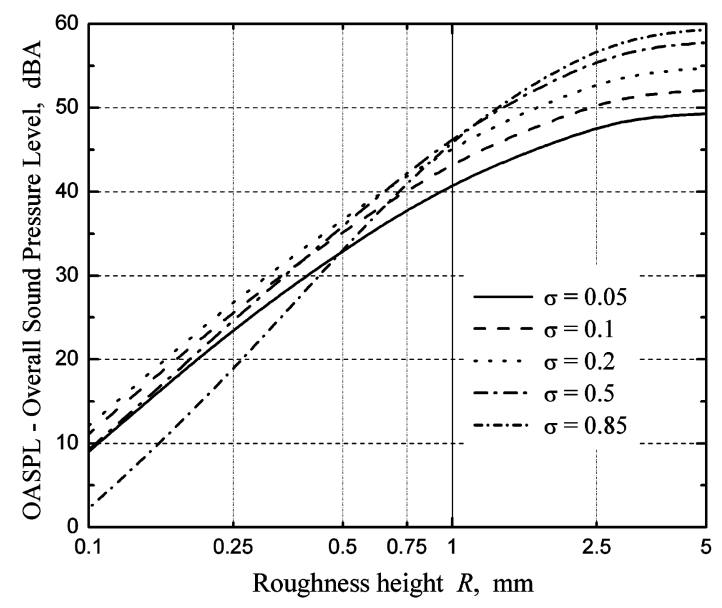

a) OASPL vs. $R$

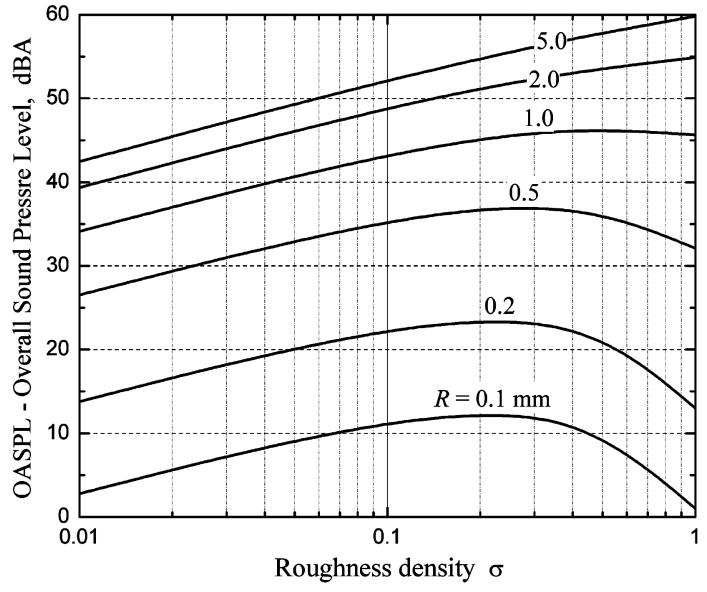

b) OASPL vs. $\sigma$

Fig. 10 Effects of roughness height $R$ and roughness density $\sigma$ on predicted roughness noise OASPL.

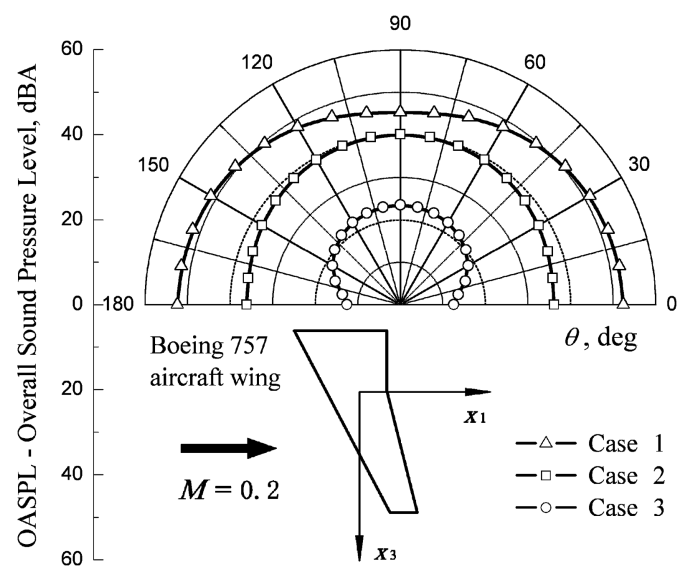

Fig. 11 Predicted directivities of roughness noise OASPL for test cases 1-3, $|x|=500 \mathrm{~m}, \phi=-\pi / 2$.

into account when $\phi \neq 0$, and it accounts for the sound from a dipole in the plane of the wing but normal to the flow direction. The integrand in $I_{3}$ shows the features of a periodic odd function of $\sin \alpha$, and thus its integration with respect to $\alpha \in[0,2 \pi]$ is identically zero.

The directivities of roughness noise OASPL in the plane of a Boeing 757 sized aircraft wing, that is, $\theta \in[0, \pi],|\phi|=\pi / 2$, were predicted for the three idealized test cases, as shown in Fig. 11. It can be seen from Eq. (41) that the roughness noise OASPL is symmetrical to $\theta=\pi / 2\left(I_{3} \equiv 0\right)$. In addition, case 1 indicates that

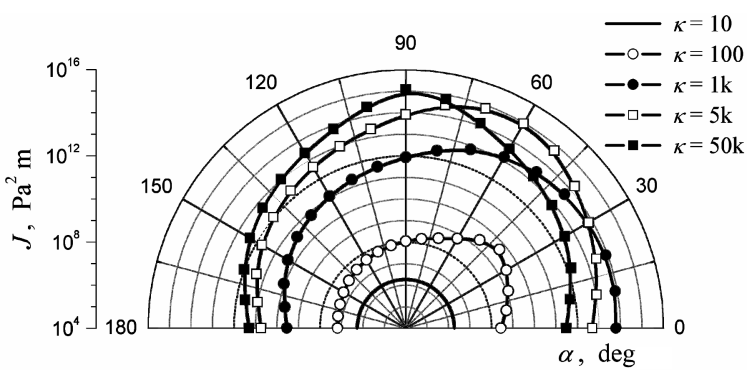

Fig. $12 J$, as defined in Eq. (61), as a function of $\kappa$ and $\alpha$.

the radiated roughness noise is $7 \mathrm{dBA}$ higher in $\theta=0, \pi$ than in $\theta=\pi / 2\left(I_{1}>I_{2}\right)$, whereas for cases 2 and 3 the maximum sound occurs in the direction $\theta=\pi / 2\left(I_{1}<I_{2}\right)$. We can understand this by looking at the variation of the integrands in Eq. (42) with $\kappa$ and $\alpha$. Figure $\underline{12}$ is a plot of the integral,

$$
J=\int_{40 \pi}^{4 \times 10^{4} \pi} 10^{C_{A} / 10}|\gamma(\kappa)|^{2} \tilde{\Phi}_{p}(\kappa, \omega) \kappa \mathrm{d} \omega
$$

as a function of $\alpha$ for different values of $\kappa . J$ needs to be multiplied by $\left(\cos ^{2} \alpha, \sin ^{2} \alpha\right) \Psi(\kappa) e^{-2 \kappa R} \delta^{* 3}$ and integrated over $\kappa \delta^{*}$ and $\alpha$ to give $\int 10^{C_{A} / 10}\left(I_{1}, I_{2}\right) \mathrm{d} \omega$, which leads to the roughness noise OASPL. As shown in Fig. 12, the variation of $J$ with the wave number direction $\alpha$ changes as $\kappa$ increases with the maximum strength moving towards $\alpha=\pi / 2$. The exponential factor $e^{-2 \kappa R}$ means that the directivity of OASPL is predominantly influenced by small $\kappa$ for large roughness 


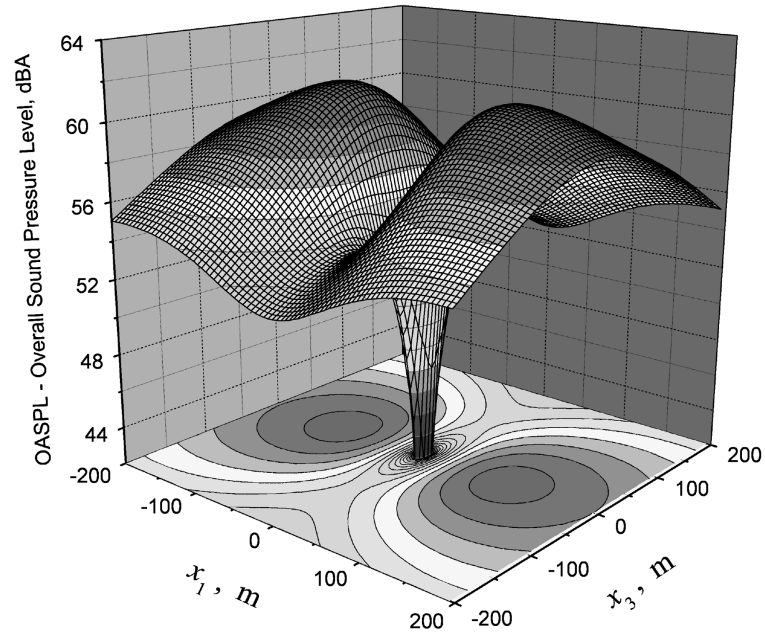

a) Case $1, R=5 \mathrm{~mm}, \sigma=0.05$

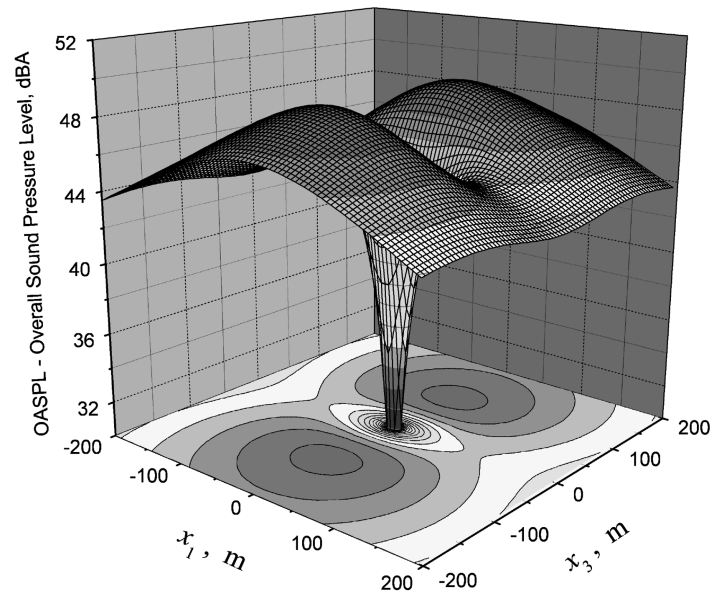

b) Case 2, $R=0.5 \mathrm{~mm}, \sigma=0.85$

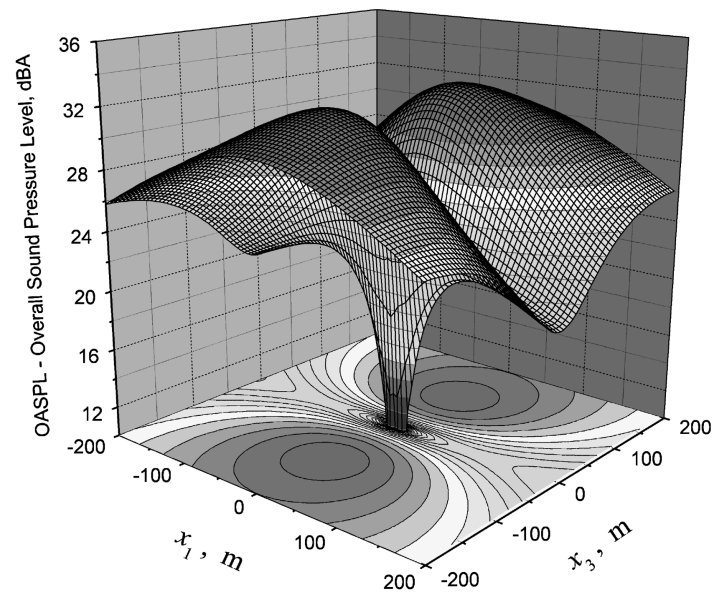

c) Case 3, $R=0.152 \mathrm{~mm}, \sigma=0.85$

Fig. 13 Predicted contours of roughness noise OASPL on the ground for test cases 1-3, $H=100 \mathrm{~m}$. height, whereas a wider range of $\kappa$ is important for small $R$. This explains the observed difference in the predicted directivities for different levels of surface roughness in Figs. 11 and 13.

In Fig. 13 the predicted contours of roughness noise OASPL on the ground are depicted in the $x_{1}-x_{3}$ coordinates for test cases $1-3$ to investigate the radiated roughness noise which can be heard by the people outside the airport perimeter in a typical built-up area. Suppose that an aircraft right above the origin $(0,0)$ at a height $H=100 \mathrm{~m}$ is approaching to or taking off from the airport in the positive $x_{1}$ direction with a Mach number $M=0.2$. In this case the roughness noise radiated from the aircraft wing to the ground should vary with the observer direction $(\theta, \phi)$, and the far-field distance $|\boldsymbol{x}|$ of an observer on the ground is also a function of $(\theta, \phi)$, that is, $|\boldsymbol{x}|=H / \sin \theta \cos \phi$.

As can be seen in Fig. 13, the predicted contours are illustrated in a $400 \times 400 \mathrm{~m}$ square region, and exhibit symmetries to both $x_{1}$ and $x_{3}$ axes as explained earlier. The contours are not shown in a central circular region of radius $5 \mathrm{~m}$ because for an observer at $(0,0)$ the observer vector $\boldsymbol{x}$ is in the direction perpendicular to both dipoles described by $I_{1}$ and $I_{2}$, namely,

$$
\begin{aligned}
\left\langle p^{2}(\boldsymbol{x}, t)\right\rangle & \rightarrow 0, \quad \text { OASPL } \\
\text { when } \quad \theta \rightarrow-\infty & \rightarrow-\infty \quad \phi \rightarrow 0
\end{aligned}
$$

Therefore the roughness noise OASPL drops abruptly as the observer approaches the origin and the lowest OASPL is observed at positions right underneath the aircraft for all test cases. However, the highest OASPL occurs on the $x_{1}$ axis for case $1\left(I_{1}>I_{2}\right)$ and on the $x_{3}$ axis for cases 2 and $3\left(I_{1}<I_{2}\right)$, which is consistent with the features of the predicted directivities in Fig. 11. Note that the highest OASPL is always positioned at a distance $H$ from the origin $(0,0)$. This is because on these axes the far-field distance $|\boldsymbol{x}|$ and directivity function $D(\theta, \phi)$ are reduced and the acoustic frequency spectrum $P_{R}(\boldsymbol{x}, \omega)$ becomes proportional to $\sin ^{2} 2 \theta$ or $\sin ^{2} 2 \phi$, as shown in Table 3 , which gives the maximum OASPL in $\theta=\pi / 4,3 \pi / 4$ on the $x_{1}$ axis or in $\phi=\pi / 4,-\pi / 4$ on the $x_{3}$ axis.

\section{Conclusions}

Howe [4] has developed a theory of sound generation by turbulent boundary-layer flow over a rough wall, in which the surface roughness is modeled by a distribution of rigid, hemispherical bosses on a rigid plane. The roughness noise is attributed to the diffraction of the turbulence near field by the bosses, and calculated by means of conventional asymptotic approximation. An empirical model was also proposed by Howe [14] by curve fitting Hersh's experimental data [15].

In this paper, the diffraction theory has been extended to numerically quantify the radiated roughness noise from a finite surface area to the far field using available models of smooth-wall wave-number-frequency spectrum scaled by the enhanced friction velocity and boundary-layer thickness for a rough surface. The objective has been to ascertain the reliability of this solution scheme and the possible importance of surface roughness to airframe noise. Acoustic measurements have been performed, and the cross spectra measured by the $\mathrm{B} \& \mathrm{~K}$ microphones have shown higher roughness noise than the noise from the smooth plate in $1-2.5 \mathrm{kHz}$ frequency. Comparisons of predicted roughness noise spectra with both experimental results and Howe's empirical model [14] have provided preliminary confirmation of the validity of the diffraction theory and solution scheme.

In the numerical prediction for the far-field radiated roughness noise of a Boeing 757 sized aircraft wing, the absolute levels of

Table 3 Reduced forms of relative expressions for observers on the $x_{1}$ and $x_{3}$ axes

\begin{tabular}{lcccccc}
\hline \hline Observer location & $\theta$ & $|\phi|$ & $|\boldsymbol{x}|$ & $D(\theta, \phi)$ & $P_{R}(\boldsymbol{x}, \omega)$ & $\mathrm{OASPL}_{\max }$ \\
\hline$x_{1}$ axis & $(0, \pi)$ & 0 & $H / \sin \theta$ & $I_{1} \cos ^{2} \theta$ & $\propto \sin ^{2} 2 \theta$ & $\theta=\pi / 4,3 \pi / 4$ \\
$x_{3}$ axis & $\pi / 2$ & {$[0, \pi / 2)$} & $H / \cos \phi$ & $I_{2} \sin ^{2} \phi$ & $\propto \sin ^{2} 2 \phi$ & $\phi=\pi / 4,-\pi / 4$ \\
\hline \hline
\end{tabular}


roughness noise SPL and OASPL for three idealized test cases have been approximately quantified. It has been shown that TE noise is only significant at very low frequencies, whereas roughness noise turns out to be dominant noise of a clean airframe in the high frequency region. The spectral level of roughness noise exceeds that of TE noise at sufficiently high frequencies, and corresponding differences in OASPL can be observed, too. Another indirect effect of surface roughness on airframe noise is accounted for by the somewhat enhanced TE noise. The subsequent parametric study has indicated that both roughness height and roughness density have significant effects on roughness noise with the former affecting the roughness noise more dominantly. In addition the directivity study has shown that the roughness noise OASPL is symmetrical to both $x_{1}$ and $x_{3}$ axes. The lowest OASPL has been observed right underneath the aircraft, and the highest OASPL occurs on the $x_{1}$ axis for case 1 and on the $x_{3}$ axis for cases 2 and 3 at the same distance from the origin as the aircraft height. In conclusion, the significant contribution of surface roughness to airframe noise needs to be carefully considered in the design of a low-noise airframe.

\section{Appendix: Models for Smooth-Wall Wave-Number- Frequency Spectrum}

The different models for the wave-number-frequency spectrum of turbulent boundary-layer pressures on a smooth wall are listed as follows. This is based on the model comparison by Graham []], but only the formulae and constant values are given for quick reference. For the sake of clarity the original notations are accordingly altered to be consistent with the definitions of this paper.

\section{The Corcos Model []}

$$
\tilde{\Phi}_{p}(\kappa, \omega)=\frac{4 \alpha_{1} \alpha_{3}}{\left[\alpha_{1}^{2}+\left(U_{c} \kappa_{1} / \omega-1\right)^{2}\right]\left[\alpha_{3}^{2}+U_{c}^{2} \kappa_{3}^{2} / \omega^{2}\right]}
$$

where $\alpha_{1}$ and $\alpha_{3}$ are parameters chosen to yield the best agreement with experiment. Various values for $\alpha_{1}$ and $\alpha_{3}$ are given in the literature; herein $\alpha_{1}=0.1$ and $\alpha_{3}=0.77$ are used as suggested by Graham [6].

\section{The Efimtsov Model [ $[\mathbf{8}]$}

$$
\tilde{\Phi}_{p}(\kappa, \omega)=\frac{\omega^{2}}{U_{c}^{2}} \frac{4 \Lambda_{1} \Lambda_{3}}{\left[1+\Lambda_{1}^{2}\left(\kappa_{1}-\omega / U_{c}\right)^{2}\right]\left[1+\Lambda_{3}^{2} \kappa_{3}^{2}\right]}
$$

where the correlation lengths, $\Lambda=U_{c} /|\omega| \alpha_{1}$ and $\Lambda_{3}=U_{c} /|\omega| \alpha_{3}$, are given by the empirical expressions

$$
\begin{array}{r}
\frac{\Lambda_{1}}{\delta}=\left[\left(\frac{a_{1} S h}{U_{c} / u_{\tau}}\right)^{2}+\frac{a_{2}^{2}}{S h^{2}+\left(a_{2} / a_{3}\right)^{2}}\right]^{-1 / 2} \\
\frac{\Lambda_{3}}{\delta}= \begin{cases}{\left[\left(\frac{a_{4} S h}{U_{c} / u_{\tau}}\right)^{2}+\frac{a_{5}^{2}}{S h^{2}+\left(a_{5} / a_{6}\right)^{2}}\right]^{-1 / 2},} & M<0.75 \\
{\left[\left(\frac{a_{4} S h}{U_{c} / u_{\tau}}\right)^{2}+a_{7}^{2}\right]^{-1 / 2},} & M>0.9\end{cases}
\end{array}
$$

where $S h=\omega \delta / u_{\tau}$ is the Strouhal number. The empirical constants $a_{1}-a_{7}$ are, respectively,

$$
\begin{gathered}
a_{1}=0.1, \quad a_{2}=72.8, \quad a_{3}=1.54, \quad a_{4}=0.77 \\
a_{5}=548, \quad a_{6}=13.5, \quad a_{7}=5.66
\end{gathered}
$$

\section{The Smol'yakov and Tkachenko Model [9]}

$$
\tilde{\Phi}_{p}(\boldsymbol{\kappa}, \omega)=0.974 A(\omega) h(\omega)[F(\kappa, \omega)-\Delta F(\boldsymbol{\kappa}, \omega)]
$$

where

$$
\begin{gathered}
A(\omega)=0.124\left[1-\frac{U_{c}}{4 \omega \delta^{*}}+\left(\frac{U_{c}}{4 \omega \delta^{*}}\right)^{2}\right]^{1 / 2} \\
h(\omega)=\left[1-\frac{m_{1} A}{6.515 \sqrt{G}}\right]^{-1} \\
m_{1}=\frac{1+A^{2}}{1.025+A^{2}}, \quad G=1+A^{2}-1.005 m_{1} \\
F(\kappa, \omega)=\left[A^{2}+\left(1-\frac{\kappa_{1} U_{c}}{\omega}\right)^{2}+\left(\frac{\kappa_{3} U_{c}}{6.45 \omega}\right)^{2}\right]^{-3 / 2} \\
\Delta F(\kappa, \omega)=0.995\left[1+A^{2}+\frac{1.005}{m_{1}}\left\{\left(m_{1}-\frac{\kappa_{1} U_{c}}{\omega}\right)^{2}\right.\right. \\
\left.\left.+\left(\frac{\kappa_{3} U_{c}}{\omega}\right)^{2}-m_{1}^{2}\right\}\right]^{-3 / 2}
\end{gathered}
$$

Here $\delta^{*}$ is the boundary-layer displacement thickness, taken as $\delta^{*}=\delta / 8$.

\section{The Ffowcs Williams Model [10]}

$$
\tilde{\Phi}_{p}(\boldsymbol{\kappa}, \omega)=\left(\frac{U_{c}|\boldsymbol{\kappa}|}{\omega}\right)^{2} \frac{4 \alpha_{1} \alpha_{3}}{\left[\alpha_{1}^{2}+\left(U_{c} \kappa_{1} / \omega-1\right)^{2}\right]\left[\alpha_{3}^{2}+U_{c}^{2} \kappa_{3}^{2} / \omega^{2}\right]}
$$

with the same values for $\alpha_{1}$ and $\alpha_{3}$ in the Corcos model.

\section{The Chase 1 Model [11]}

$$
\begin{gathered}
\tilde{\Phi}_{p}(\boldsymbol{\kappa}, \omega)=\frac{\rho_{0}^{2} \omega^{2} u_{\tau}^{3}}{U_{c}^{2} \Phi(\omega)}\left(\frac{C_{M} \kappa_{1}^{2}}{\left[K_{+}^{2}+\left(b_{M} \delta\right)^{-2}\right]^{5 / 2}}\right. \\
\left.+\frac{C_{T}|\kappa|^{2}}{\left[K_{+}^{2}+\left(b_{T} \delta\right)^{-2}\right]^{5 / 2}}\right)
\end{gathered}
$$

where

$$
\begin{gathered}
K_{+}^{2}=\frac{\left(\omega-U_{c} \kappa_{1}\right)^{2}}{h^{2} u_{\tau}^{2}}+|\kappa|^{2} \\
\frac{\Phi(\omega)}{2 \pi}=\frac{2 \pi h \rho_{0}^{2} u_{\tau}^{4}}{3 \omega\left(1+\mu^{2}\right)}\left(C_{M} F_{M}+C_{T} F_{T}\right) \\
F_{T}=\frac{1+\mu^{2} \alpha_{M}^{2}+\mu^{4}\left(\alpha_{M}^{2}-1\right)}{\left[\alpha_{M}^{2}+\mu^{2}\left(\alpha_{M}^{2}-1\right)\right]^{3 / 2}} \\
{\left[\alpha_{T}^{2}+\mu^{2}\left(\alpha_{T}^{2}-1\right)\right]^{3 / 2}}
\end{gathered}
$$

$$
\alpha_{M \text { or } T}^{2}=1+\left(U_{c} / b_{M \text { or } T} \omega \delta\right)^{2}, \quad \mu=h u_{\tau} / U_{c}
$$


Based on Chase's recommendations, the empirical constants have the following values:

$$
\begin{gathered}
C_{M}=0.0745, \quad C_{T}=0.0475, \quad b_{M}=0.756 \\
b_{T}=0.378, \quad h=3.0
\end{gathered}
$$

\section{The Chase 2 Model [12]}

$$
\begin{gathered}
\tilde{\Phi}_{p}(\boldsymbol{\kappa}, \omega)=\frac{\rho_{0}^{2} \omega^{2} u_{\tau}^{3}}{U_{c}^{2} \Phi(\omega)\left[K_{+}^{2}+(b \delta)^{-2}\right]^{5 / 2}} \\
\times\left(C_{M} \kappa_{1}^{2}+C_{T}|\boldsymbol{\kappa}|^{2} \frac{K_{+}^{2}+(b \delta)^{-2}}{|\kappa|^{2}+(b \delta)^{-2}}\right)
\end{gathered}
$$

where

$$
\begin{gathered}
K_{+}^{2}=\frac{\left(\omega-U_{c} \kappa_{1}\right)^{2}}{h^{2} u_{\tau}^{2}}+|\kappa|^{2} \\
\frac{\Phi(\omega)}{2 \pi}=\frac{2 \pi h \rho_{0}^{2} u_{\tau}^{4}}{3 \omega\left(1+\mu^{2}\right)}\left(C_{M} F_{M}+C_{T} F_{T}\right) \\
F_{M}=\frac{1+\mu^{2} \alpha^{2}+\mu^{4}\left(\alpha^{2}-1\right)}{\left[\alpha^{2}+\mu^{2}\left(\alpha^{2}-1\right)\right]^{3 / 2}} \\
F_{T}=\frac{3\left(1+\mu^{2}\right)\left(1+\alpha^{2}\right)}{2 \alpha^{3}} \\
\alpha^{2}=1+\left(U_{c} / b \omega \delta\right)^{2}, \quad \mu=h u_{\tau} / U_{c}
\end{gathered}
$$

The recommended empirical constant values are

$$
b=0.75, \quad h=3.0, \quad h C_{M}=0.466, \quad h C_{T}=0.014
$$

\section{Acknowledgments}

This work is part of a collaborative project, Silent Aircraft Initiative (SAI), funded by the Cambridge-MIT Institute (CMI), whose support is gratefully acknowledged. The authors would also like to thank the Overseas Research Studentship (ORS) and Gates Cambridge Scholarship, which have made this research possible. Moreover, special thanks go to Ho-Chul Shin for kind assistance to the acoustic measurements, Will Graham for advice on the form of the smooth-wall pressure spectrum, and Andrew Faszer for helpful discussions on the TE noise models.

\section{References}

[1] Faszer, A., "Effect of Boundary Layer Manipulation on Airframe Trailing-Edge Noise," Ph.D. Thesis, Dept. of Engineering, Univ. of Cambridge, Cambridge, England, 2007.

[2] Franzoni, L., "Assessment of the Contribution of Panel Vibration to Airframe Noise," AIAA Journal, Vol. 42, No. 8, Aug. 2004, pp. 15221528.

[3] Schlichting, H., Boundary Layer Theory, 7th ed., McGraw-Hill, New York, 1979.

[4] Howe, M. S., "On the Generation of Sound by Turbulent Boundary Layer Flow over a Rough Wall," Proceedings of the Royal Society of
London, Vol. A395, No. 1809, Oct. 1984, pp. 247-263.

[5] Howe, M. S., "The Influence of Viscous Surface Stress on the Production of Sound by a Turbulent Boundary Layer over a Rough Wall," Journal of Sound and Vibration, Vol. 104, No. 1, 1986, pp. 29 39.

[6] Graham, W. R., "A Comparison of Models for the WavenumberFrequency Spectrum of Turbulent Boundary Layer Pressures," Journal of Sound and Vibration, Vol. 206, No. 4, 1997, pp. 541-565.

[7] Corcos, G. M., "The Structure of the Turbulent Pressure Field in Boundary-Layer Flows," Journal of Fluid Mechanics, Vol. 18, No. 3, 1964, pp. 353-378.

[8] Efimtsov, B. M., "Characteristics of the Field of Turbulent Wall Pressure Fluctuations at Large Reynolds Numbers," Soviet Physics Acoustics, Vol. 28, No. 4, 1982, pp. 289-292.

[9] Smol'yakov, A. V., and Tkachenko, V. M., "Model of a Field of Pseudosonic Turbulent Wall Pressures and Experimental Data," Soviet Physics Acoustics, Vol. 37, No. 6, 1991, pp. 627-631.

[10] Ffowcs Williams, J. E., "Boundary-Layer Pressures and the Corcos Model: a Development to Incorporate Low Wavenumber Constraints," Journal of Fluid Mechanics, Vol. 125, Dec. 1982, pp. 9-25.

[11] Chase, D. M., "Modeling the Wavevector-Frequency Spectrum of Turbulent Boundary Layer Wall Pressure," Journal of Sound and Vibration, Vol. 70, No. 1, 1980, pp. 29-67.

[12] Chase, D. M., "The Character of the Turbulent Wall Pressure Spectrum at Subconvective Wavenumbers and a Suggested Comprehensive Model," Journal of Sound and Vibration, Vol. 112, No. 1, 1987, pp. $125-147$.

[13] Howe, M. S., "The Turbulent Boundary-Layer Rough-Wall Pressure Spectrum at Acoustic and Subconvective Wavenumbers," Proceedings of the Royal Society of London, Vol. A415, No. 1848, Jan. 1988, pp. 141-161.

[14] Howe, M. S., Acoustics of Fluid-Structure Interactions, Cambridge Univ. Press, Cambridge, England, 1998.

[15] Hersh, A. S., "Surface Roughness Generated Flow Noise," AIAA Paper 83-0786, 1983.

[16] Mills, A. F., and Hang, X., "On the Skin Friction Coefficient for a Fully Rough Flat Plate," Journal of Fluids Engineering, Vol. 105, No. 3, Sept. 1983, pp. 364-365.

[17] Blake, W. K., "Turbulent Bounday-Layer Wall-Pressure Fluctuations on Smooth and Rough Walls," Journal of Fluid Mechanics, Vol. 44, No. 4, Dec. 1970, pp. 637-660.

[18] Schultz, M. P., and Flack, K. A., "Outer Layer Similarity in Fully Rough Turbulent Boundary Layers," Experiments in Fluids, Vol. 38, No. 3 , March 2005, pp. 328-340.

[19] Crighton, D. G., Dowling, A. P., Ffowcs Williams, J. E., Heckl, M., and Leppington, F. G., Modern Methods in Analytical Acoustics: Lecture Notes, Springer-Verlag, London, 1992.

[20] Blake, W. K., Mechanics of Flow-Induced Sound and Vibration, Vol. 2: Complex Flow-Structure Interactions, Academic Press, New York, 1986.

[21] Anon., "The Mean Skin Friction Coefficient for a Rough Flat Plate with a Turbulent Two-Dimensional Boundary Layer in Compressible Adiabatic Flow, with Application to Wedges, Cylinders and Cones," ESDU Aerodynamics Data Item 2c-73016, 1973.

[22] Prandtl, L., and Schlichting, H., "Resistance Law of Rough Plates," Werft-Reederei-Hafen, Vol. 15, No. 1, 1934, pp. 1-4.

[23] Nikuradse, J., "Laws of Flow in Rough Pipes," NACA TM 1292, 1950.

[24] von Kármán, T., "Turbulence and Skin Friction," Journal of the Royal Aeronautical Society, Vol. 1, No. 1, 1934, pp. 1-20.

[25] Krogstad, P. A., Antonia, R. A., and Browne, L. W. B., "Comparison Between Rough- and Smooth- Wall Turbulent Boundary Layers," Journal of Fluid Mechanics, Vol. 245, Dec. 1992, pp. 599-617.

[26] Ahn, B., "Modeling Unsteady Wall Pressures beneath Turbulent Boundary Layers," Ph.D. Thesis, Dept. of Engineering, Univ. of Cambridge, Cambridge, England, 2005.

[27] Duncan, W. J., Thom, A. S., and Young, A. D., Mechanics of Fluids, 2nd ed., Edward Arnold, London, 1970.

[28] Dowling, A. P., and Ffowcs Williams, J. E., Sound and Sources of Sound, Ellis Horwood, Chichester, England, 1983.
C. Bailly

Associate Editor 